Submission for Publication in International Journal of Impact Engineering (Special Issue for Experimental Testing and Computational Modeling)

\title{
Explicit Phantom Paired Shell Element Approach for Crack Branching and Impact Damage Prediction of Aluminum Structures
}

\author{
Jim Lua $^{*(a)}$, Tingting Zhang ${ }^{(a)}$, Eugene Fang ${ }^{(a)}$, and Jeong-Hoon Song ${ }^{*(b)}$ \\ Correspondence to: \\ jlua@gem-innovation.com (Jim Lua, Senior Principal Scientist) \\ jh.song@colorado.edu (Jeong-Hoon Song, Assistant Professor) \\ (a) Global Engineering and Materials, Inc. \\ 1 Airport Place, Suite 1, Princeton, NJ 08540

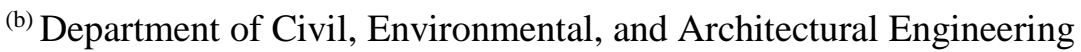 \\ Univeristy of Colorado, Boulder, CO 80309
}

\section{Abstract}

The main objective of this paper is to exploit an efficient and a simplified extended finite element shell formulation for prediction of ductile fracture and crack branching of thin-walled aluminium structures subjected to impact loading. In order to characterize an arbitrary crack initiation, propagation and brunching, a phantom paired shell element approach is further developed and implemented into Abaqus' explicit solver via its user defined element (VUEL). The energy dissipation due to failure is captured by a cohesive force along the crack interface when its accumulative plastic strain reaches a critical value. A numerical technique for modelling out-of-plane crack branching phenomena is also developed by activating the phantom nodes and re-grouping the element connectivity. Four numericcal examples are used to demonstrate the applicability and accurcy of the extended shell element apporch for ductile failue predictoion of an indentaion test, a multi-bay stiffened panel with crack branching, an explosively loaded plate, and a cylinder subjected to impulsive loading.

(1)


Submission for Publication in International Journal of Impact Engineering (Special Issue for Experimental Testing and Computational Modeling)

\section{Introduction}

21 The use of aluminum alloys and their extruded structural components in advanced vehicle construction has

22 a great advantage in minimizing a vessel's weight. Given its one-third density of steel and 70\% of the tensile

23 strength of steel, the resulting weight of aluminum high speed vehicle is much lower than a similar vessel

24 constructed from steel. The reduction of structural weight allows for increased payload, top speed, and

25 operation range at lower operational and total ownership cost. Since a vehicle is an operation-critical

26 structure, it must be designed to have a sufficient level of residual strength both during and after an extreme

27 loading event such as impact. The extreme dynamic loading can seriously damage the vessel form by

28 dishing and holing the stiffened panel. To ensure the sufficient strength and damage tolerance of a structural

29 component, a full-scale analysis coupled with experimentation has to be implemented during the design

30 iteration, structural certification, and shock qualification process.

32 Design of large, high-speed aluminum vessels operating in a hostile environment requires the welded structure to withstand sub-critical growth of manufacturing flaws and service-induced defects against failure under extreme dynamic loading. The compounding effects from material heterogeneity and reduced strength in Heat Affect Zone (HAZ) makes the welded aluminum structure prone to crack initiation under normal operating conditions. In the presence of unexpected extreme loading events, these initial flaws will

37 propagate, branch, and be arrested by the nearby stiffeners. The complexity in component geometry, material heterogeneity, and 3D stress distribution, will likely make crack growth curvilinear, turning towards the stiffener. Failure to account for the 3D crack growth geometry may lead to poor predictions of the crack tip driving force which will have a pronounced impact on the fracture pattern and its resulting

41 load-deflection prediction.

43 Typical size of a stiffened panel used in a large vehicle structure is about $10 \mathrm{~m} \times 10 \mathrm{~m}$ with a thickness of $44 \quad 0.013 \mathrm{~m}$. For such thin-walled structures, the computational effort using solid finite elements is prohibitive 
Submission for Publication in International Journal of Impact Engineering (Special Issue for Experimental Testing and Computational Modeling)

if a good element aspect ratio is maintained. Structural shell elements are widely used in such applications

because they offer the advantage of allowing the use of large elements while maintaining adequate

47

numerical accuracy. Typical shell elements have an in-plane size of 2 to 30 times larger than the plate thickness. It is therefore highly desirable to develop an efficient fracture analysis and design tool to characterize crack growth simulation in a large scale thin-walled structure within an efficient shell element formulation.

When the crack path is unknown a priori, a pioneering approach based on cohesive elements embedded at all element boundaries is developed by Xu and Needleman (1994) and the method had been improved by Camacho and Ortiz (1996) by introducing cohesive elements only along element boundaries where a certain fracture criterion is met. Given the pre-defined element topology, the crack growth direction is expected to be mesh dependent in addition to the added artificial compliance in the model resulting from the finite cohesive stiffness used.

In order to characterize an arbitrary crack initiation and propagation, the extended finite element method (XFEM) coupled with shell kinematics was developed by Arias, Song and Belytschko (2005) based on the Mindlin-Reissner theory and an enhanced assumed strain formulation to alleviate locking in thin shells. Instead of using the approach of nodal enrichment via additional degrees of freedom, an alternative approach based on two overlapping elements has been developed by Hansbo and Hansbo (2004) where their identical kinematical representation to XFEM has been proved by Song et al. (2006). A phantom paired approach coupled with a cohesive injection has been developed by Song and Belytschko (2009) and implemented for the Belytschko-Tsay (B-T) shell element (Belytschko and Tsay, 1984). To further enhance the computational efficiency, a one-point integration scheme along with element-wise progression of the crack is implemented by Song and Belytschko (2009) for the simulation of dynamic cracks in thin shells and its applications to quasi brittle fracture problems. Note that the partition of unity enriched methods for static and dynamic fracture in thin shell analysis have been also developed by Rabczuk et al. (2006, 2007, 
Submission for Publication in International Journal of Impact Engineering (Special Issue for Experimental Testing and Computational Modeling)

71 2010), Chau-Dinh et al. (2012), and Nguyen-Thanh et al. (2015) besides from the alternative methods for

72 fracture in thin shells by Areias and Rabczuk (2013) and Amiri et al. (2014). A 7-parameter, 6-node

73 triangular solid-shell element in conjunction with a rate-dependent cohesive zone model has been developed

74 by Larsson et al. (2011) and enhanced by Mostofizadeh (2013) by implementing an explicit time stepping

75 scheme, the Johnson Cook phenomenological model, and a shifted cohesive zone model. However, the

76 implementation of these XFEM methodologies for shell elements is still within a standalone finite element

77 code with limited capabilities in terms of element library, material constitutive models, contact algorithms,

78 and graphic user interface for finite element model generation and display of analysis results.

80 Driven by the strong needs from commercial industries and DoD Labs in the application of commercial

81 finite element software such as Abaqus for the design and certification of large scale thin-walled metallic

82 structures, it is imperative to implement the XFEM for shell elements within a commercial finite element

83 software. A great challenge exists since a commercial finite element software such as Abaqus has placed a

84 restriction in altering the number of elements and their connectivity. The presence of multiple cracks and

85 their growths in different planes will make the kinematic description of these cracks challenging within an

86 XFEM framework for Abaqus. A novel approach for characterization of multiple crack initiations and

87 propagations has to be developed via the phantom nodes re-grouping and re-assignment of element 88 connectivity.

90 The present paper is divided into five sections. In Section 2, the development of phantom paired

91 Belytschko-Tsay (B-T) shell (Belytschko and Tsay 1984) is presented for Abaqus along with its numerical

92 implementation. Section 3 describes the modeling approach for crack branching along with its capability

93 demonstration. Section 4 gives the description of a material model and a crack initial and propagation

94 criterion. The final demonstration is reported in Section 5, in which four examples are selected for the

95 ductile failure simulation of an indentation test, a multi-bay stiffened panel with crack branching, an

96 explosively loaded square plate, and a cylinder subjected to impulsive loading. 
Submission for Publication in International Journal of Impact Engineering (Special Issue for Experimental Testing and Computational Modeling)

\section{Phantom paired Belytschko-Tsay (B-T) shell for Abaqus}

\subsection{Overview of a $B-T$ shell}

The B-T shell is based on a combined co-rotational and velocity strain formulation. The co-rotational

101

102

103

104

105

106

107

108

109

110

111

112

113

114

portion of the formulation avoids the complexities by embedding a coordinate system in the element. The selection of velocity-strain or rate-of-deformation in the B-T shell formulation makes the conjugate stress the same as the physical Cauchy stress that can greatly facilitate the constitutive description.

The velocity field of the shell is given by

$$
\mathbf{v}(\xi, t)=\mathbf{v}^{\text {mid }}(\xi, t)-\varsigma \mathbf{e}_{3} \times \boldsymbol{\theta}^{\text {mid }}(\boldsymbol{\xi}, t)
$$

where $\mathbf{v}^{\text {mid }} \in R^{3}$ are the velocities of the shell mid-surface, $\boldsymbol{\theta}^{\text {mid }} \in R^{3}$ are angular velocities of the normal to the mid-surface, $\varsigma$ is the pseudo thickness which varies linearly from $-h / 2$ to $h / 2$ along the shell thickness $h$, and $\xi=\left(\xi_{1}, \xi_{2}\right)$ are material coordinates of the manifold that describes the mid-surface of the shell. The nomenclature used in Eq. (1) is illustrated in Fig. 1.

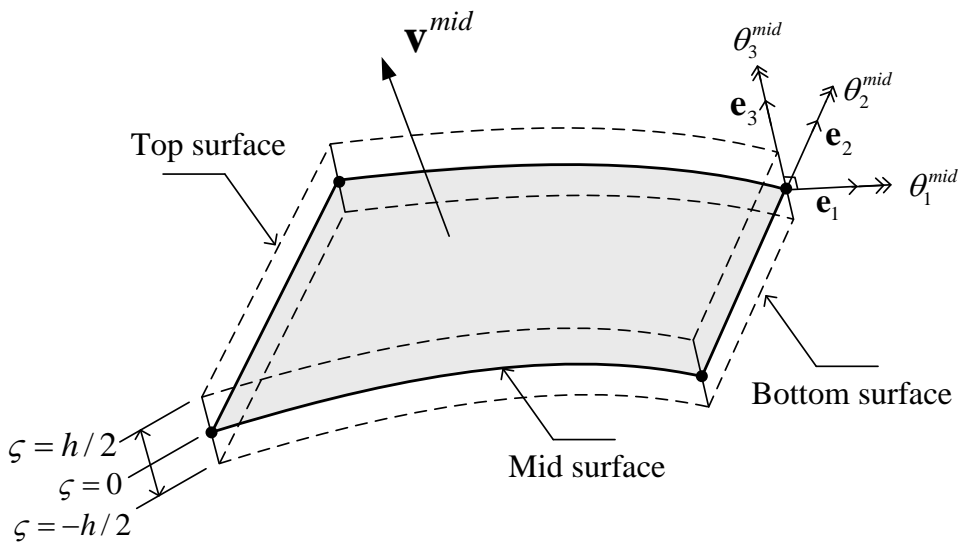

Figure 1 A geometric description of Belytschko-Tsay shell 
Submission for Publication in International Journal of Impact Engineering (Special Issue for Experimental Testing and Computational Modeling)

115 A kinematic theory based on corotational rate-of-deformation and corotational Cauchy stress rate is used.

116 The corotational coordinate system serves as a local coordinate system to measure the rate-of-deformation

117 of elements during the simulations as shown in Fig. 2.

118

119

120

121

122

123

124

125

126

127

128

129

130 After substituting Eq. (2-3) into Eq. (2-2), we have defined as

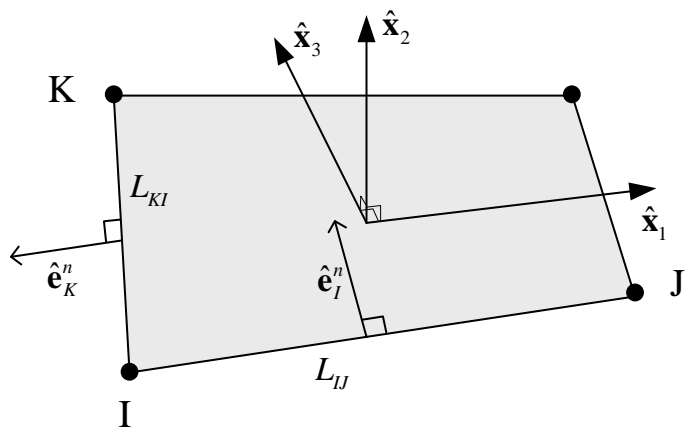

Figure 2 Vector and edge definitions for the transverse shear projection scheme

Using Eq. (1), the velocity components of an arbitrary point with thickness-direction coordinate can be

$$
\begin{aligned}
& v_{x}=v_{x}^{\text {mid }}+\theta_{y}^{\text {mid }} \varsigma, \\
& v_{y}=v_{y}^{\text {mid }}-\theta_{x}^{\text {mid }} \varsigma, \\
& v_{z}=v_{z}^{\text {mid }}
\end{aligned}
$$


Submission for Publication in International Journal of Impact Engineering (Special Issue for Experimental Testing and Computational Modeling)

$$
d_{x}=\frac{\partial v_{x}^{m i d}}{\partial x}+\varsigma \frac{\partial \theta_{y}^{m i d}}{\partial x}
$$

$$
2 d_{x z}=\frac{\partial v_{z}^{m i d}}{\partial y}+\theta_{y}^{m i d}
$$

The rate form of the constitutive law expressed in corotational system can be written by

$$
\dot{\sigma}=C(t, \sigma, d, \ldots) \dot{d}
$$

142 where $\dot{\sigma}$ is the Cauchy stress rate and $C$ is the fourth-order material tensor, which, in general, may depend 143 upon time, $t$, stress, strain rate, back stress if plasticity is involved. The components of the velocity strain, 144 i.e., Eq. (4), can be directly substituted into Eq. (5) to evaluate the corresponding stress tensor components. 145 The stress and velocity-strain components are conjugate in the sense that they can be used in a principle of 146 virtual power for the explicit element formation.

\subsection{Formulation of a phantom paired B-T shell}

149 In order to represent mesh-independent crack propagation in cracked shell elements, a phantom paired 150 element approach originally proposed by Hansbo and Hansbo (2004) is selected for its Abaqus 151 implementation. As shown in Fig. 3, a kinematic description of a cracked shell can be represented by a pair 
Submission for Publication in International Journal of Impact Engineering (Special Issue for Experimental Testing and Computational Modeling)

of elements. Each of these two elements has two physical nodes (solid circles) and two phantom nodes representation is given by

$$
u(X, t)=\sum_{I=1}^{n^{N}} N_{I}(X)\left\{u_{I}(t)+q_{I}\left[H(f(X))-H\left(f\left(X_{I}\right)\right)\right]\right\}
$$

157

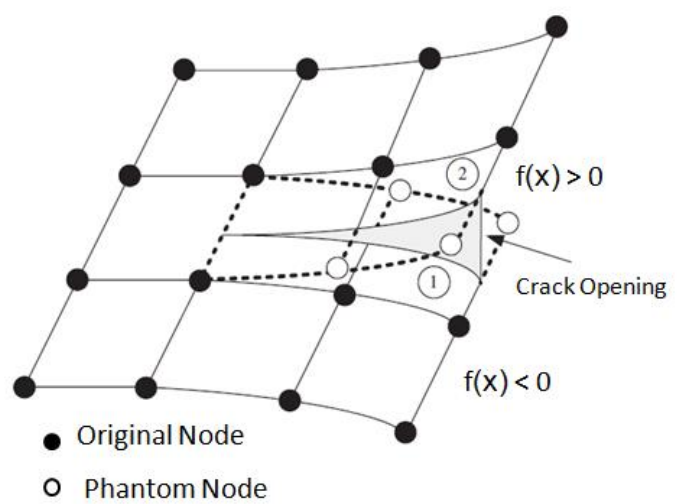

Figure 3 Representation of a cracked shell using two 4-node elements: 2 solid and 2 hollow circles denote the original nodes and the added phantom nodes, respectively.

where $u_{I}$ is the standard degree of freedoms (DoFs), $q_{I}$ is the enriched DoFs and $H$ is the Heaviside function. The signed distance function $f$ indicates on which side of the crack the concerned position is. Expanding the above by subdividing each term into parts that are associated with $f(X)<0$ and $f(X)>0$, we have

By defining

$$
u=\sum_{I=1}^{n^{N}}\left[u_{I} N_{I}(1-H)+u_{I} N_{I} H+q_{I}\left(H-H_{I}\right) N_{I}\right]
$$


Submission for Publication in International Journal of Impact Engineering (Special Issue for Experimental Testing and Computational Modeling)

$$
\begin{aligned}
& u_{I}^{1}= \begin{cases}u_{I} & \text { if } f\left(X_{I}\right)<0 \\
u_{I}-q_{I} & \text { if } f\left(X_{I}\right)>0\end{cases} \\
& u_{I}^{2}= \begin{cases}u_{I}+q_{I} & \text { if } f\left(X_{I}\right)<0 \\
u_{I} & \text { if } f\left(X_{I}\right)>0\end{cases}
\end{aligned}
$$

173 We can rewrite Eq. (7) as

$$
u(X, t)=\sum_{I \in S_{1}} u_{I}^{1} \underbrace{(t) N_{I}(X)}_{u^{1}(X, t)} H(-f(X))+\sum_{I \in S_{2}} u_{I}^{2} \underbrace{(t) N_{I}(X)}_{u^{2}(X, t)} H(f(X))
$$

where $S_{1}$ and $S_{2}$ are the index sets of the nodes of superposed elements 1 and 2, respectively, shown in Fig. 3 for the case of a 4-node B-T shell. Because of the use of the one-point integration based on the fixed Gauss quadrature point, no projection needs to be made to map the history variables for the subdomain integration associated with an evolving crack.

Without adding any new nodes along the crack line, the two mathematical elements are separated after the connectivity rearrangement. Thus, the total displacement field for a completely cut element can be represented by the sum of two element fields: $u^{1}(X, t)$, which holds for $f(X)<0$ and $u^{2}(X, t)$, which defines for $f(X)>0$. Recasting the discontinuous field in this form simplifies the implementation of the element in an existing finite element code such as Abaqus. It is only necessary to activate extra elements (i.e. Element

1872 in this case) and modify the corresponding element connectivity to describe an arbitrary cracked shell 188 element.

Both the linear and angular velocities of the shell mid-surface $\left(v^{\text {mid }}, \theta^{\text {nid }}\right)$ have to be decomposed first into 191 its continuous and discontinuous part during its XFEM formulation. The discontinuity of a field variable 192 across a crack surface that is normal to the mid-surface of the shell can be represented equivalently via a 
Submission for Publication in International Journal of Impact Engineering (Special Issue for Experimental Testing and Computational Modeling)

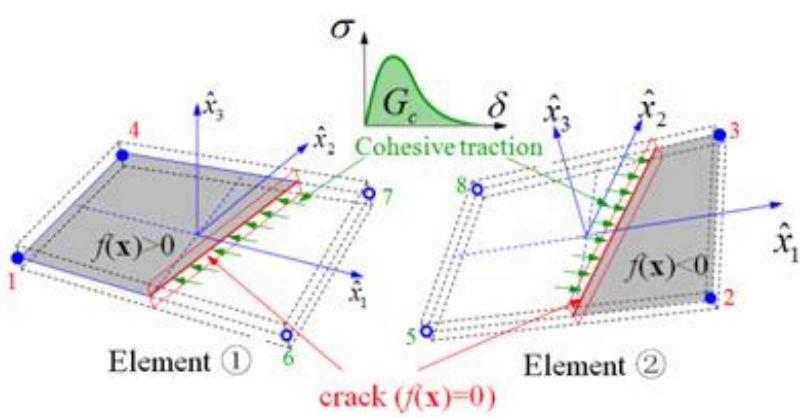

Figure 4 Illustration of XFEM formulation for a cracked Belytschko-Tsay shell

A cohesive interaction is embedded along a newly cracked surface where the energy dissipation is captured from its initial opening to a fully cracked stage as shown in Fig. 4. It is assumed that a crack in a shell is always through its thickness and normal to the mid surface of the shell. In addition, during the dynamic crack growth, the tips are always in an element edge resulting in a completely cut element. Using the 204 principal of virtual power, the nodal forces $\left\{f^{\text {int }}, f^{x x t}, f^{k i n}, f^{\text {coh }}\right\}$ are computed based on the internal work $205\left(\delta W^{\text {int }}\right)$, the external work performed by applied load $\left(\delta W^{\text {ext }}\right)$, the kinetic work performed by inertia force $206\left(\delta W^{k i n}\right)$, and the work performed by the cohesive traction on the crack surface $\Gamma_{c}$ during its opening $\left(\delta W^{c o h}\right)$, 207 respectively. A final system of equations can be formulated based on the balance of linear momentum 208 equation. A discretized element formulation is constructed by introducing a shape function and one point 209 integration scheme. Using the one-point integration based on the fixed Gauss quadrature point, no 210 projection needs to be made to map the history variables for the subdomain integration associated with an 211 evolving crack. With the phantom paired element approach, the nodal force vectors associated with a 212 cracked element can be computed using the reference nodal force without a crack multiplied by its 
Submission for Publication in International Journal of Impact Engineering (Special Issue for Experimental Testing and Computational Modeling)

213

214

215

216

217

218

219

associated area ratio of phantom element 1, i.e., $\left(\mathrm{A}_{\mathrm{e} 1} / \mathrm{A}_{0}\right)$, or $\mathrm{A}_{\mathrm{e} 2} / \mathrm{A}_{0}$ for element 2 . The same approach can used to compute the nodal mass of a cracked element.

\subsection{Implementation of phantom paired B-T shell via Abaqus VUEL}

Since Abaqus does not allow the user to either alter the DoFs or insert new elements during an analysis, overlay elements are pre-defined in the XFEM zone (VUEL zone) by doubling the nodes associated with each user-defined element. The VUEL routine, the user element in Abaqus/Explicit, has to be developed to define the contribution of the element to the internal or external force/flux vector, form the mass/capacity matrix, and update the solution-dependent state variables associated with the element. At each call of VUEL, Abaqus/Explicit provides the values of the nodal coordinates and of all solution-dependent nodal variables (displacements, velocities, accelerations, etc.) at all degrees of freedom associated with the element, as well as values of the solution-dependent state variables associated with the element at the beginning of the current increment.

In VUEL, the element nodal force $\left(F^{J}\right)$ has to be computed from the values of the nodal variables $u^{M}$, the rate of nodal variables $\dot{u}^{M}$, and the solution-dependent state variables $H$ within the element. The solutiondependent state variables can include the damage driven constitutive parameters and predefined field variables. The mass is computed only once at the beginning of the analysis and it is not allowed to be changed within the VUEL during the solution process.

The implementation of the phantom paired shell elements in Abaqus is accomplished via the overlay elements defined over the user-defined elements zone. When a user-defined element is in its primitive state without a crack, only its real element is activated while its phantom part is muted. In this case, all nodes of the real element are real (or physical) and they have independent DoFs determined by the explicit analysis. All nodes of the phantom element are phantom (or unphysical) and their field variables are not calculated 
Submission for Publication in International Journal of Impact Engineering (Special Issue for Experimental Testing and Computational Modeling)

but rather inherited from the real element. This inheritance of field variables from real element to phantom element is very critical because it prepares the phantom element for future calculations when the physical element is cut resulting in the activation of the phantom element.

242 Figure 5(a) shows a logic diagram for the implementation of a cracked shell in Abaqus' explicit via its 243 VUEL. During the first call of VUEL, a lamped nodal mass will be defined and the subsequent change of 244 the nodal mass matrix is not allowed. The internal nodal force vector $\left(\hat{f}_{e}^{\text {it }}\right.$ is computed for each subelement 245 (Element 1-2-7-8 and Element 5-6-3-4) where the contribution of each subelement to the total internal force of the cracked shell element is scaled by $\alpha_{i}$ defined by the ratio of the activated area to the total area of the

247 element. In addition, both the translational and rotational mass matrix have to be scaled for the pair of overlay elements based on the relative position between the crack front and element edge. Therefore, the element mass matrix in VUEL needs to be changed based on the position of the crack cut position.

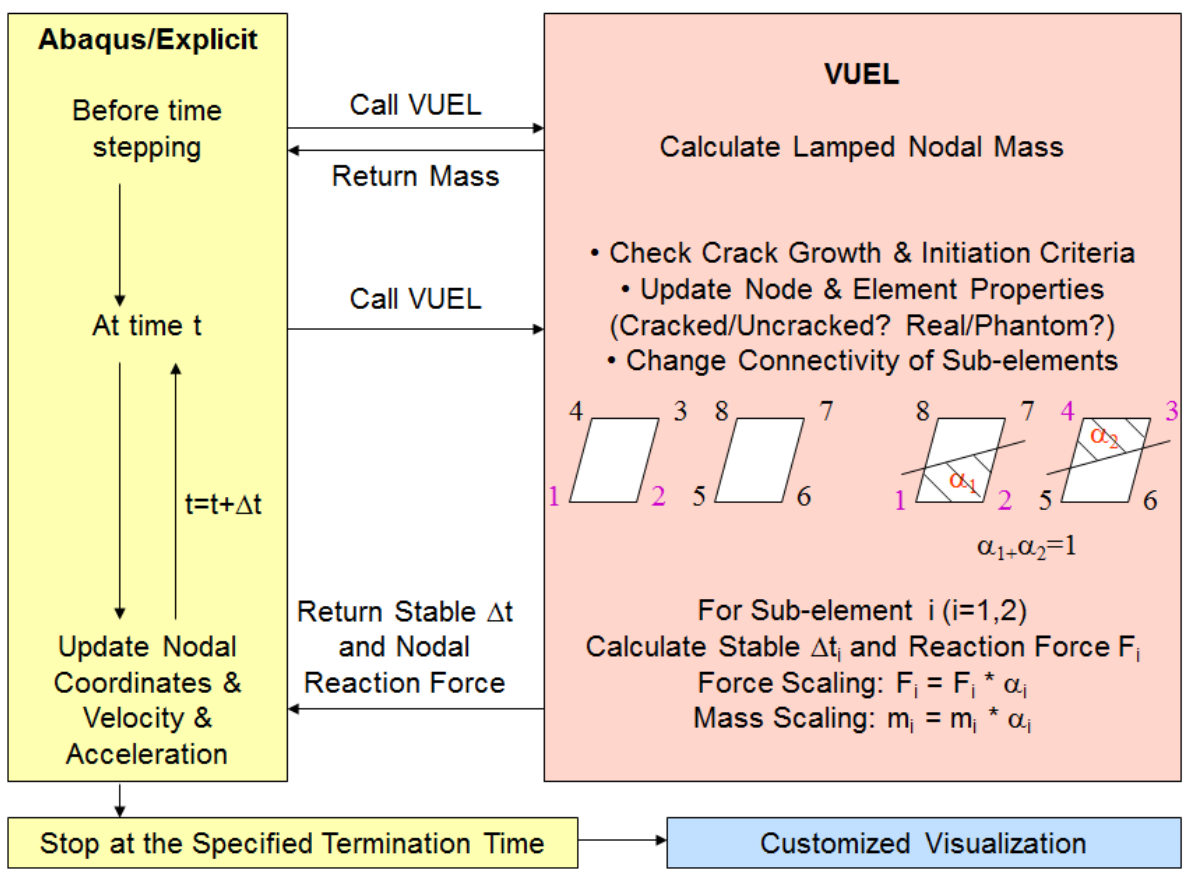


Submission for Publication in International Journal of Impact Engineering (Special Issue for Experimental Testing and Computational Modeling)

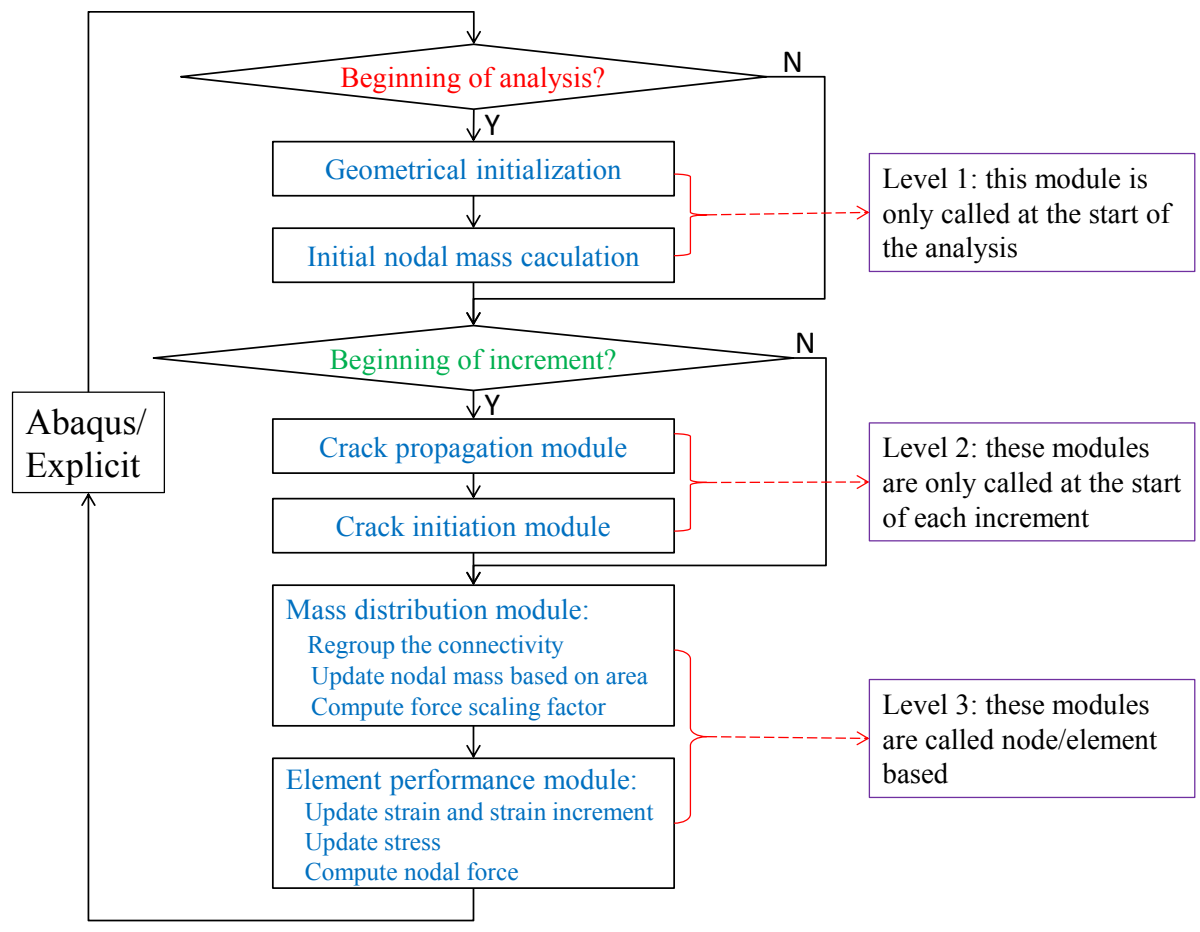

(b)

Figure 5 Implementation of a phantom paired shell elements (XSHELL) in Abaqus via its VUEL: (a) Logic diagram of the VUEL developed for XSHELL and (b) its flow chart for the implementation.

When a crack extends to an XFEM element, the element connectivity and mass associated with both the real and phantom elements have to be changed. Unlike wake cracked elements, a tip element is a cracked element where the crack tip resides on one of the element edges. Under the assumption of element-byelement crack growth, the crack tip is always on an element edge, and no tip enrichment or slicing is required during numerical integration. For a tip element, the aforementioned element reconstruction rule has to be modified. The two physical nodes associated with the edge where the crack tip resides on must be shared by both the real and phantom elements. An illustration of this special treatment is shown in the inset of Fig. 6. Assuming the crack tip resides on the left edge defined by nodes 1 and 2, both the real and

267 phantom elements share edge 1-2; this ensures that at the crack tip, the crack is closed (i.e. crack opening 268 displacement equals to zero). 
Submission for Publication in International Journal of Impact Engineering (Special Issue for Experimental Testing and Computational Modeling)

270 For the implementation, the XSHELL program is called by Abaqus/Explicit in three levels as shown in

271 Figure 5(b). In Level 1, the program reads in the control parameters and input data associated with mesh

272 and cracks. The geometry information is used to initialize relevant global variables and determine the nodal

273 mass. At the analysis increment level, XSHELL will check whether or not the program is called at the

274 beginning of an increment. If the answer is true, XSHELL will execute two separate geometry update

275 analysis modules. The first module is to check if any of the elements ahead of current cracks has reached

276 the critical conditions and update the crack configuration if necessary. The other one is to check if failure

277 criterion is satisfied in the elements which are away from current cracks. A new crack will be initiated from

278 the elements that satisfy the failure criterion. After updating the cracked geometry resulting from the new

279 crack initiation and propagation of existing cracks, the nodes of elements affected by the geometry change

280 will be regrouped to form the phantom paired elements. Correspondingly, relevant nodal mass needs to be

281 recomputed based on the area associated with split element fragments. An element level analysis consists

282 of three parts, i.e., the displacement field approximation to obtain the strain, the use of a material

constitutive model to update the stress and virtual work principle to compute the equivalent nodal force.

284 Since Abaqus/Explicit only accepts the nodal mass at the beginning of analysis, all the nodal mass

the nodal force based on the ratio of redistributed nodal mass to its initial nodal mass and compute the scaled nodal force within Abaqus/Explicit solver to achieve a consistent nodal acceleration.

When an element is split into two (both real and phantom elements are activated), the mass has to be redistributed based on the area of the physical sub-domain (shaded area in Fig. 4) included in the real or 291 phantom element representation. An illustration of the mass allocation rule that has been implemented is 292 given in Fig. 7 for a stationary crack (Case 1), a growing crack (Case 2), and crack initiation in the middle 293 followed by its propagation at both ends (Case 3). For illustrative purposes, a unit mass is assumed for each 294 of the physical elements, and the re-distributed nodal mass is annotated below the nodal number. In the 295 case of crack initiation followed by propagation (Case 3), the two elements ahead of each crack tip are 
Submission for Publication in International Journal of Impact Engineering (Special Issue for Experimental Testing and Computational Modeling)

intact before the crack propagates, so only real elements are activated and they both take the full mass of the physical elements; as such, each node of these two real elements (nodes 1-4 and 9-12) is assigned a quarter of the mass of the physical element, i.e. 1/4.

For the three elements in the middle (Case 3), their phantom elements are activated and each of them takes half the mass of the physical element, which is then divided by the four nodes composing the phantom element. Quantitatively, the real and phantom elements associated with physical elements 2-4 all have a mass of $1 / 2$ each; the nodes composing these elements are then equally assigned a quarter of the element mass, i.e. 1/8. To compute the total nodal mass, the attributes from all elements sharing the node are summed up. For example, node 4 takes $1 / 4$ from the uncracked element 1 and 1/8 from the real element of element 2 ; that is $3 / 8$ in total. After crack propagation, Element 5 is cracked so its phantom element is activated and takes half the mass of the element. Comparing the nodal mass before and after crack nodes 25-28.
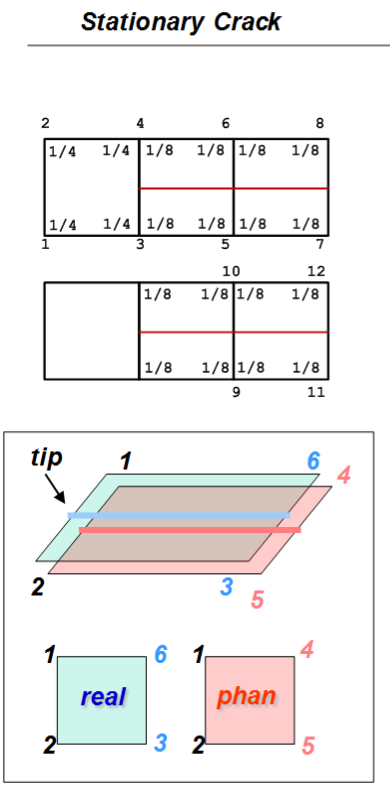
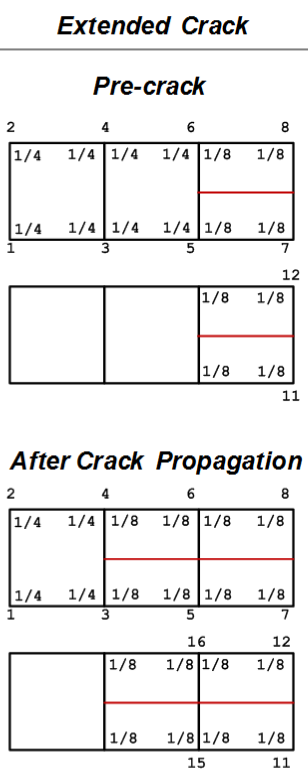

Newly Initiated Crack

After Crack Initiation
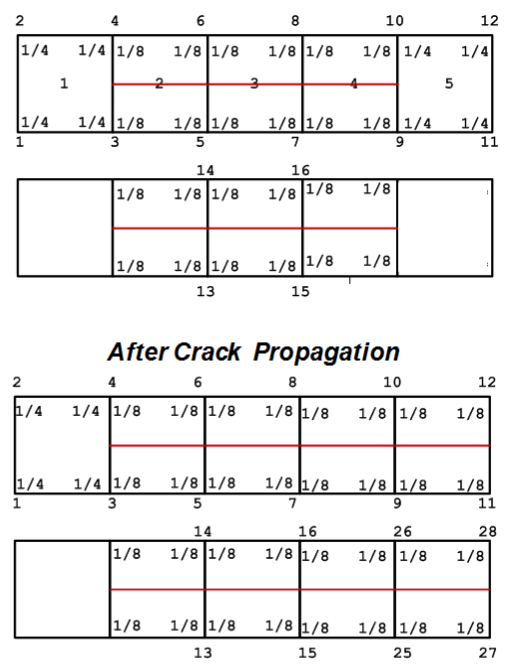
Submission for Publication in International Journal of Impact Engineering (Special Issue for Experimental Testing and Computational Modeling)

Figure 6 Change of element connectivity and mass allocation for three representative case scenarios of simple cracking (where opposite edges are cracked).

\section{Modeling of crack branching}

As described in Section 2.3, distinct treatments have been implemented for the tip and internal elements based on the relative position between the crack and the element boundary. When a crack meets at an intersection of multiple planes with distinct orientation, a crack branching phenomena has to be explicitly addressed for a correct kinematic representation of a cracked geometry. A great challenge to model the crack branching is how to numerically identify the intersection zone, welded or intersected element and introduce the additional crack tips after branching. A node-wise search is performed at the beginning of the

322 simulation to identify the edges that are shared by more than two elements. An overview of this crack 323 branching algorithm is illustrated in Fig. 7 and 8. Two geometric identities that play a key role in the crack 324 branching algorithm are: weld edge and weld element. The weld edges are those edges shared by more than contain at least one weld edge, such as element $e_{1}, e_{2}$ and $e_{3}$ in Fig. 7.

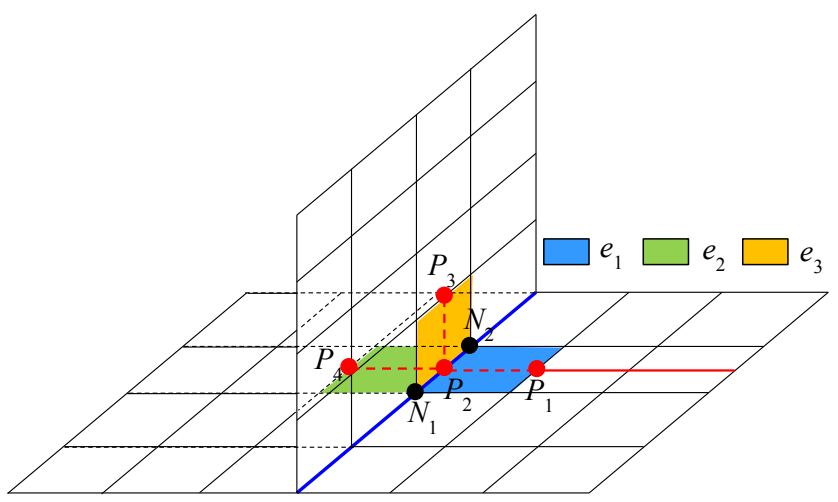


Submission for Publication in International Journal of Impact Engineering (Special Issue for Experimental Testing and Computational Modeling)

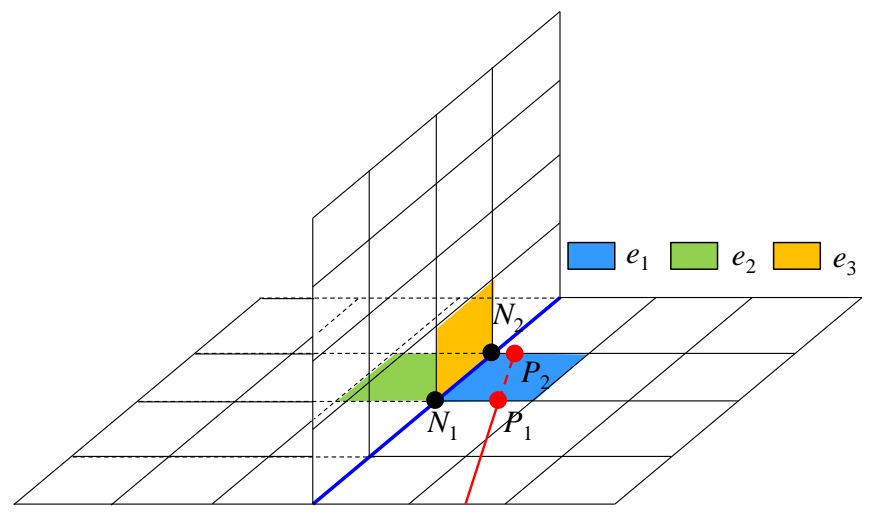

Figure 8 The exceptional case under current crack branching algorithm

The crack branching algorithm is summarized as follows:

1) Check if the current tip element is a weld element. If it is not, the crack moves forward without branching. If the tip element is a weld element and satisfies failure criterion as the element $e_{1}$ shown in Fig. 7, move to Step 2.

2) Check if the new crack tip is located in the weld edge (now it is $N_{2} N_{3}$ shown in Fig. 9. If the crack tip $P_{w}$ is on the weld edge, move to Step 3; If not, the crack moves forward without branching;

3) Set the two neighboring weld elements $\left(e_{2}\right.$ and $\left.e_{3}\right)$ as the new tip elements;

4) Cut the two new tip elements based on the crack propagation direction calculated from the local stress/strain fields. Two crack tips will be obtained as $P_{3}$ and $P_{4}$;

5) If $P_{3}$ and/or $P_{4}$ are on an edge which shares nodes with the weld edge (as indicated as the $P_{4}$ in Fig. 10, modify the crack tip location from the adjacent side to the weld edge's opposite side. This modification is made for simplifying the connectivity regrouping for the cracked elements. In the case shown in Fig. 11, the phantom node of $N_{2}$ shall be activated for crack $P_{1} P_{2} P_{w} P_{3}$, but not for crack branch $P_{1} P_{2} P_{w} P_{4}$. The tip edge where $P_{4}$ is located in shares the node $N_{2}$. To bypass the contradiction, the modified crack segment $P_{w} P_{4}$ is set to be perpendicular to the weld edge in the local coordinates of $e_{2}$ so that both nodes of the weld edge will be activated once branching occurs;

6) Further crack propagation from $P_{3}$ and $P_{4}$ is the same as any crack propagation within a panel without stiffeners. 
Submission for Publication in International Journal of Impact Engineering (Special Issue for Experimental Testing and Computational Modeling)

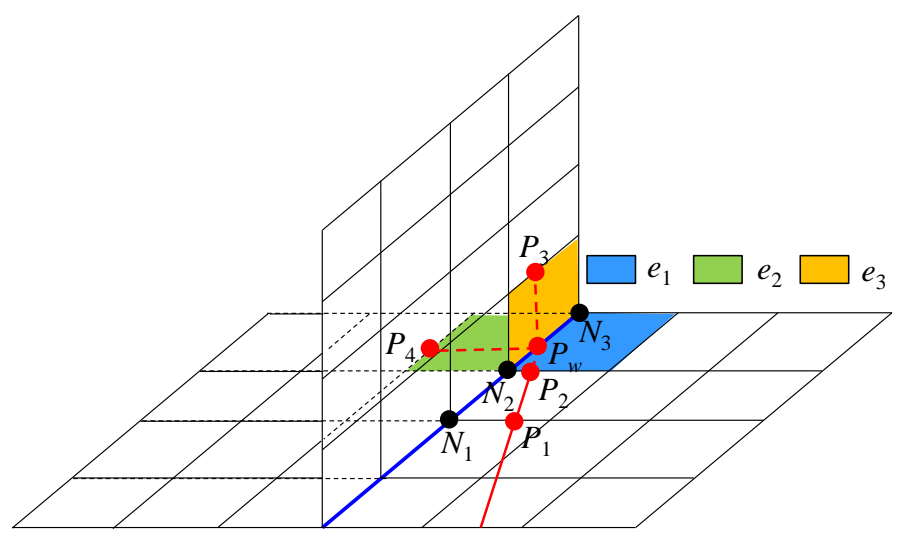

Figure 9 The enhanced crack branching algorithm

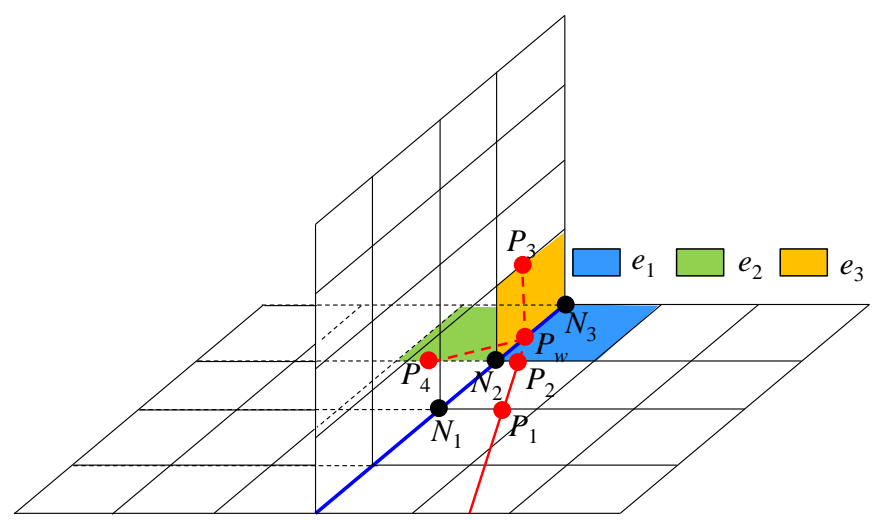

Figure 10 Demonstration of contradictory node activation $\left(\mathrm{N}_{2}\right)$ after crack branching algorithm

\section{Summary of material model and failure characterization}

\subsection{Material model}

Two approaches have been used extensively in characterizing the ductile fracture, namely, the micromechanical approach and the macroscopic approach. In the micromechanical approach, the ductile fracture is characterized via void nucleation, growth, shearing, and coalescence (Gurson (1977); Tvergaard and Needleman (1984); Nahshon and Hutchinson (2008)). While the micromechanical approach can correlate the evolution of the micro-damage with the observed physics, it requires a large number of 
Submission for Publication in International Journal of Impact Engineering (Special Issue for Experimental Testing and Computational Modeling)

be in the order of $\sim 10-100 \mathrm{~m}$ which is much smaller than the desired size of a shell element used for the structural representation of a large scale thin-walled structure.

The macroscopic approach aims at the globally measurable change of the material response due to damage material (Johnson and Cook (1985); Bai and Wierzbicki (2008); Xue et al. (2009); Gao et al. (2009); Gao et al. (2011)). The effect of the length scale is smeared via a mesh dependent calibration process. To

372 alleviate mesh dependency, the mesh used at the coupon level during the material calibration has to remain 373 the same for developing a simulation model for a large scale structure. The damage variable is determined 374 via a calibration process using experimental data. The advantage of the macroscopic approach is its 375 computational efficiency. And because of this, we have used the macroscopic approach for the material and 376 failure models used in the current approach. The most commonly used $J_{2}$ plasticity model is implemented 377 in the XFEM toolkit for shell element (XSHELL). The $J_{2}$ plasticity is assumed to present the undamaged material response in which the von Mises yield function of the materials is related only to the second stress deviator invariant $J_{2}$. For shell structure applications, the von Mises yield surface with associated plastic flow is used within the plane stress framework. An isotropic hardening behavior is assumed in MAT24 of XSHELL. No volumetric plastic strain is considered in this model.

The yield condition is given by

$$
F=\sigma_{e}-\bar{\sigma}\left(\bar{\varepsilon}^{p}\right)=0
$$

$$
\sigma_{e}=\left(\frac{3}{2} s_{i j} s_{i j}\right)^{\frac{1}{2}}=\sqrt{3 J_{2}}
$$

With the assumption of isotropic hardening, the yield stress, $\bar{\sigma}$ can be defined as a tabular function of 
Submission for Publication in International Journal of Impact Engineering (Special Issue for Experimental Testing and Computational Modeling)

slope of the tabular data is used to calculate the yield stress value for plastic strains exceeding the last value given in the tabular data. This data input format is convenient for users since the measured test data can be

392 directly used without performing the curve-fit.

\subsection{Crack initiation based on a weighted average for accumulative plastic strain}

An average accumulative plastic strain criterion is implemented in XSHELL toolkit. Based on the average accumulative plastic strain criterion, a strong discontinuity ahead of the previous crack tip is injected along the direction according to the maximum principle tensile strain direction of an averaged strain $\boldsymbol{\varepsilon}^{\text {avg }}$ when the strain at the crack tip material point reaches a fracture threshold. Such an element-wise calculation can be mesh dependent. To alleviate this issue, a non-local approach based on a weighted average is implemented to compute an averaged strain, $\boldsymbol{\varepsilon}^{a v g}$ prior to the application of a damage initiation criterion. in Fig. 11.

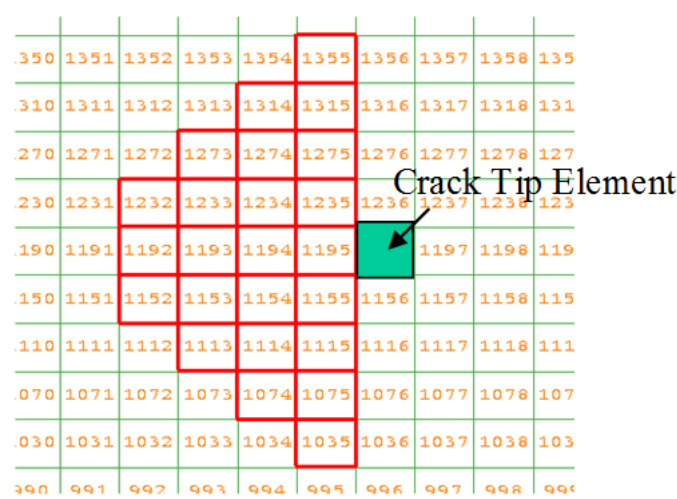

(a)

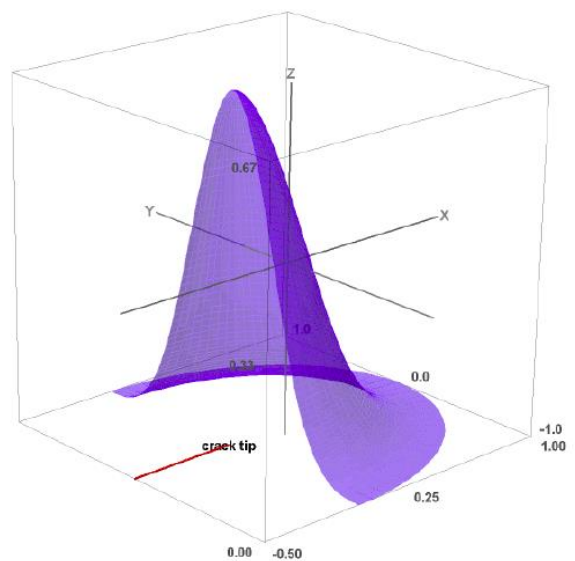

(b)

Figure11 Summary of a weighted average approach: (a) schematic of average domain; and (b) definition of a 
Submission for Publication in International Journal of Impact Engineering (Special Issue for Experimental Testing and Computational Modeling)

409

410

411

412

413

414

415

416

417

418

419

420

421

422

6

$$
w=\frac{1}{(2 \pi)^{3 / 2} l^{3}} \exp \left(-\frac{r^{2}}{2 l^{2}}\right)
$$

where $w$ is the weight, $l$ determines the interaction decay profile, and $r$ is the distance to the tip. Using Eq.

(13), the average strain, $\boldsymbol{\varepsilon}^{\text {avg }}$, can be computed by

$$
\varepsilon^{a v g}=\frac{4}{\pi} \int_{-\pi / 2}^{\pi / 2} \int_{0}^{r_{c}} w(r) \varepsilon d r d \theta
$$

where $r$ and $\theta$ are the distance from the tip and the angle formed between the position vector from the tip and the tangent to the crack path at the tip, respectively, and $w(r)$ is the weight function defined by Eq. (13). Using a cubic spline approximation, we have

423

424 For an XSHELL mesh with a relatively small element size, an empirical value for $r_{c}\left(\approx 3 h_{t i p}\right)$ can be used,

425 where $h_{t i p}$ is the size of the crack tip element. After the discretization, a pointwise calculation of Eq. (14)

426 can be performed using

427 
Submission for Publication in International Journal of Impact Engineering (Special Issue for Experimental Testing and Computational Modeling)

$$
\boldsymbol{\varepsilon}^{a v g}=\frac{\sum_{\alpha} w\left(r_{\alpha}\right) \boldsymbol{\varepsilon}_{\alpha}}{\sum_{\alpha} w\left(r_{\alpha}\right)}
$$

In Eq. (16), $w\left(r_{\alpha}\right)$ is the weight associated with point $\alpha, r_{\alpha}$ is the distance from point $\alpha$ to the crack tip,

431 and $\boldsymbol{\varepsilon}_{\alpha}$ is the strain. The parameter $r_{\alpha \sqsubseteq}$ serves as the length scale during this averaging process. With a

432 user-selected non-local zone size of $r_{c}$, all the elements within the half-circular disk (see Fig. 11) can be 433 extracted for the pointwise calculation using Eq. (16).

\subsection{Insertion of near tip cohesive zone model}

436 If the crack opening displacement is not governed by a cohesive model, the stress component normal to the

437 crack surface suddenly drops to zero after cracking without any fracture energy dissipation. As a result, 438 excessive elastic energy accumulates in the system and this will erroneously accelerate crack propagation. 439 In order to correctly account for the energy dissipation from the crack opening and its subsequent growth, 440 a cohesive injection has to be implemented once a crack initiation along a given direction has been detected. 441 The use of the cohesive injection can also alleviate the spurious mesh-dependent pathological behavior by 442 providing a bounded solution at the crack tip.

444 Right at the instant of the crack initiation, a normal traction across a potential crack surface can be 445 determined. With a user-defined fracture toughness $G_{f}$, a cohesive law can be activated to compute the 446 degradation of the traction force based on the crack opening displacement. Using a linear cohesive mode 447 as an illustration, a representation of energy dissipation during the crack opening and growth is described 448 in Fig. 12. 
Submission for Publication in International Journal of Impact Engineering (Special Issue for Experimental Testing and Computational Modeling)

450

451

452

453

454

455

456

457

458

459

460

461

462

463

464

465

466

467

$$
\mathbf{f}_{e}^{c o h}=\mathbf{R}_{e 1}^{T} \int_{\Gamma_{c}}-N^{T} \tau_{c}\left(\delta_{n}\right) \hat{\mathbf{n}} \mathrm{d} \Gamma+\mathbf{R}_{e 2}^{T} \int_{\Gamma_{c}} N^{T} \tau_{c}\left(\delta_{n}\right) \hat{\mathbf{n}} \mathrm{d} \Gamma
$$

Figure 12 Representation of a tip process zone via a cohesive model

As shown in Fig. 12, the dissipated energy due to the crack propagation is equivalent to the fracture energy:

$$
G_{f}=\frac{1}{2} \tau_{\max } \delta_{\max }
$$

where $G_{f}$ is the fracture energy, $\tau_{\max }$ and $\delta_{\max }$ are the maximum cohesive traction and the maximum crack opening displacement, respectively. Since $\tau_{\max }$ can be computed at failure and $G_{f}$ is a given material property for a material, the value of $\delta_{\max }$ can be determined from Eq. (17). The normal crack opening displacement $\left(\delta_{n}\right)$ and the resulting cohesive force in phantom pair elements are given by

$$
\delta_{n}=\hat{\mathbf{n}} \cdot\left(\hat{\mathbf{u}}^{e 2}(\xi, t)-\hat{\mathbf{u}}^{e 1}(\xi, t)\right)_{\xi \in \Gamma_{c}}
$$

where $\hat{\mathbf{n}}$ is the normal to the crack surface. The discretized form of the cohesive force vector is computed by

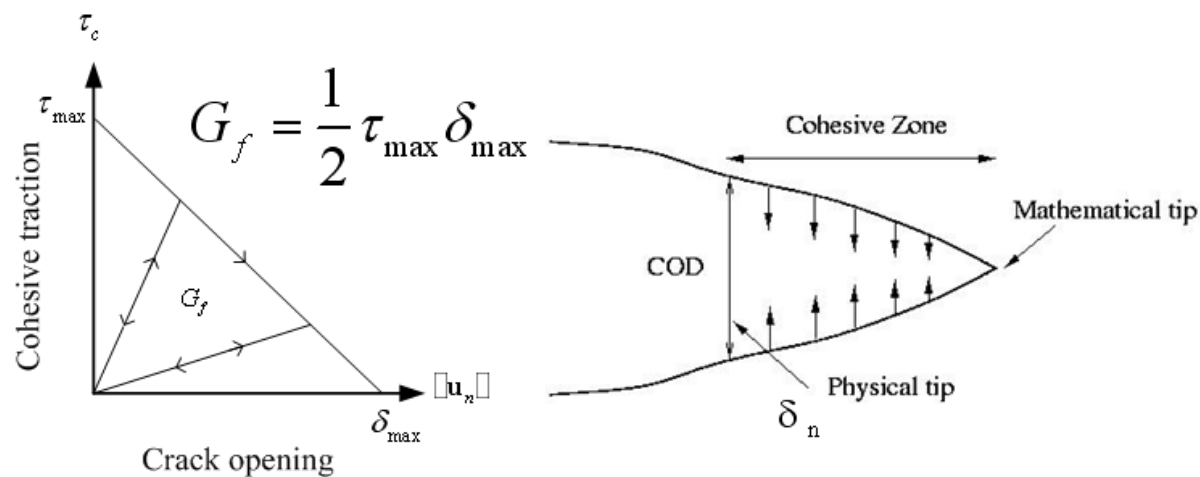


Submission for Publication in International Journal of Impact Engineering (Special Issue for Experimental Testing and Computational Modeling)

469 To illustrate the effect cohesive injection on the crack growth prediction, a plate with the thickness and

470 density of 1 is subject to a uniaxial stretching is analyzed. Five integration points are used through the plate

471 thickness. All the properties are defined in a dimensionless space. The material is assumed to be elastic-

472 plastic with piecewise plastic hardening. The Young's modulus is 1000 and the Poisson's ratio is 0.3 . Plastic

473 strain and flow stress are tabulated in Table 1 . The critical plastic strain is set to be 0.05 . The critical energy

474 release rate is 190. An initial edge crack with the length of 0.25 is embedded at the right edge of the plate,

475 along the symmetric axis in the horizontal direction. The crack extends when the prescribed displacement

476 reaches a critical value. Due to the symmetric nature of the problem, the crack propagates under Mode I

477 until the entire plate is cut through. Fig. 13 plots the pulling force versus time for cases with and without

478 the use of the cohesive model, respectively. It is evident that in the absence of the cohesive model, the load

479 response shows considerable fluctuations. With the embedded cohesive zone model, however, the load

480 response becomes much smoother. This demonstrates that the use of the cohesive model is very effective

481 in alleviating dynamic oscillation in explicit crack extension simulations. What is also shown in Fig. 13 is

482 the accumulation of cohesive energy over time.

483

Table 1 Piecewise linear hardening parameters

\begin{tabular}{|l|l|l|l|l|l|l|l|l|}
\hline Plastic Strain & 0 & 0.02 & 0.04 & 0.08 & 0.14 & 0.24 & 0.4 & 0.8 \\
\hline Stress & 100 & 200 & 300 & 400 & 500 & 600 & 700 & 800 \\
\hline
\end{tabular}


Submission for Publication in International Journal of Impact Engineering (Special Issue for Experimental Testing and Computational Modeling)
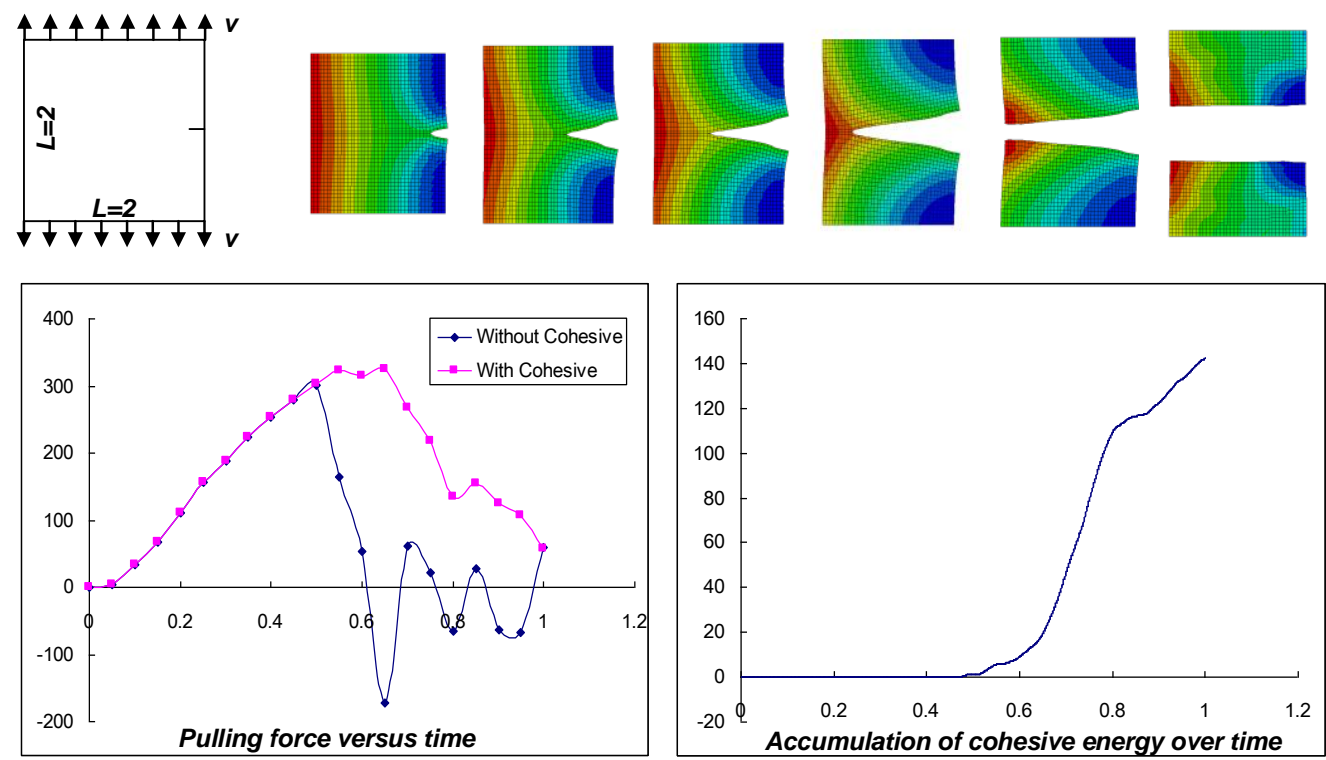

Figure 13 Numerical example of cohesive model

\subsection{Numerical Examples}

\subsection{Indentation tests without and with stiffeners}

This problem was selected based on the indentation tests conducted by Alsos and Amdahl (2009) and Alsos et al. (2009) for an unstiffened and a stiffened steel panel. The size of the steel panels is selected based on the 1:3 scale of the dimensions found in medium sized tankers. The plate length and width is $1200 \mathrm{~mm}$ and $720 \mathrm{~mm}$, respectively and the plate thickness is $5 \mathrm{~mm}$. For a flat bar stiffened panel, the two flat bar stiffeners are equally spaced with the spacing of $240 \mathrm{~mm}$. As shown in Fig. 14, the steel panel is welded to a strong frame made by four massive steel box beams. The box beam has the height of $200 \mathrm{~mm}$, width of $300 \mathrm{~mm}$, and thickness of $12.5 \mathrm{~mm}$. For the stiffened panel, the stiffeners are welded to the plate and their ends are

497 fixed to the frame.

The indenter has a cone shape with a spherical nose. The frame section is made of high strength steel (S355NH EN10210), while the flat bar stiffeners and the plate are made of mild steel (S235JR EN10025).

501 The motion of the indenter is controlled at a constant rate of $10 \mathrm{~mm} / \mathrm{min}$ which represents a quasi-static 
Submission for Publication in International Journal of Impact Engineering (Special Issue for Experimental Testing and Computational Modeling)

loading condition. A crack initiates when the indenter displacement reaches a critical value $\left(\delta_{c r}\right)$ and extends until the entire structure fails. The presence of material nonlinearity, contact, large deformation, stiffness degradation caused by crack extension, and crack opening dependent cohesive interaction are strongly coupled together in these two simulations.

To simulate the observed fracture pattern and the measured load-displacement curve shown in Fig. 14, XSHELL models are developed as shown in Fig. 15 for both the unstiffened and stiffened plate. The number of nodes and elements for the unstiffened model is 12174 and 12384 , respectively. For the model with stiffeners, the number nodes and elements in the FEA model is 16598 and 16800, respectively. Given the unknown location for the crack initiation and its subsequent crack path, the entire model is characterized using XSHELL elements for both the unstiffened and stiffened panel. The $J_{2}$ plasticity (Section 4.1) is used with its Young's modulus of 2.06e5 MPa and Poisson's ratio of 0.3. The initial yield stress for this material is $285 \mathrm{MPa}$ and a failure strain for the crack initiation is set as 0.396 .
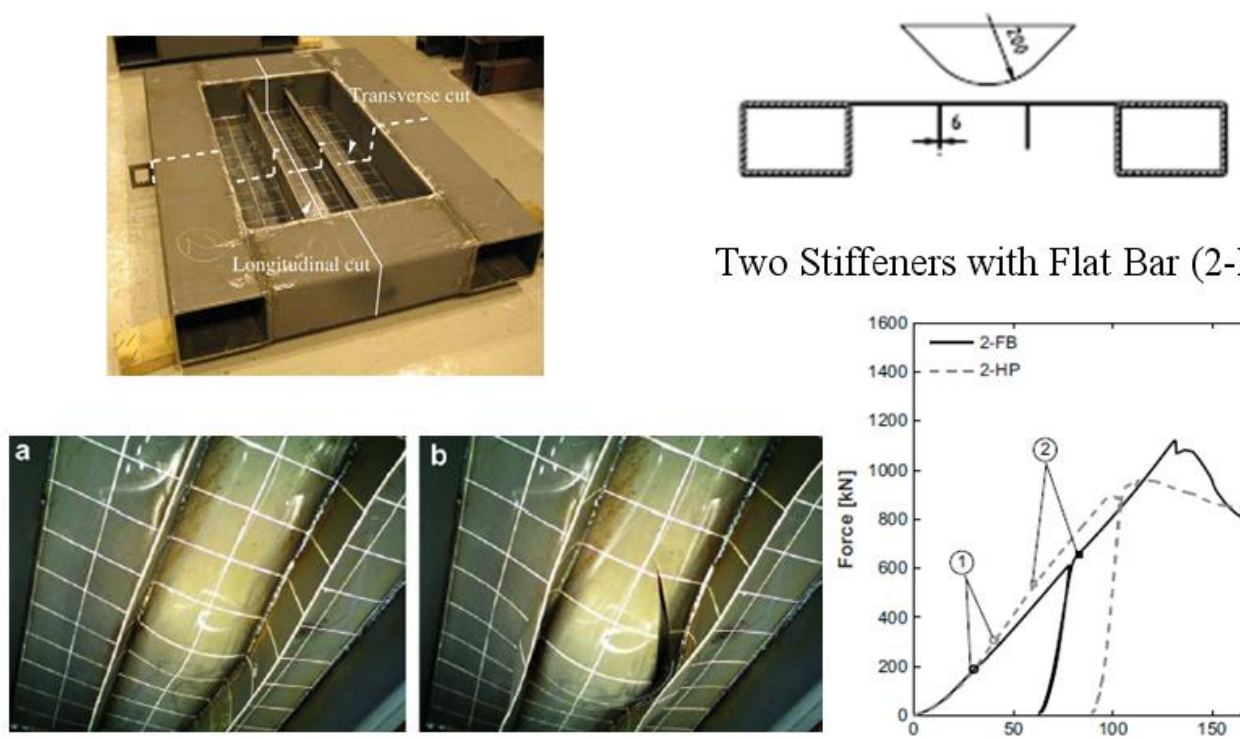

Two Stiffeners with Flat Bar (2-FB)

Figure 14 Illustration of an indentation test of a stiffened panel with observed failure pattern and load-displacement 
Submission for Publication in International Journal of Impact Engineering (Special Issue for Experimental Testing and Computational Modeling)

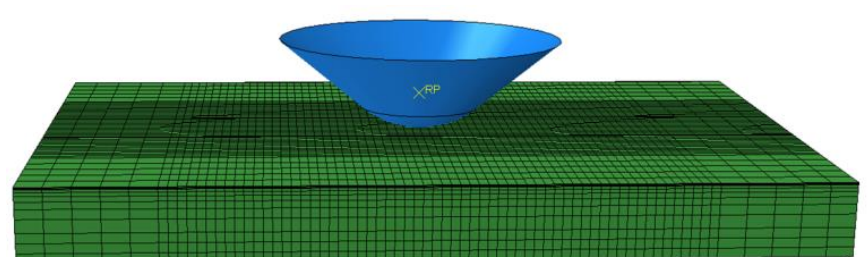

bottom view

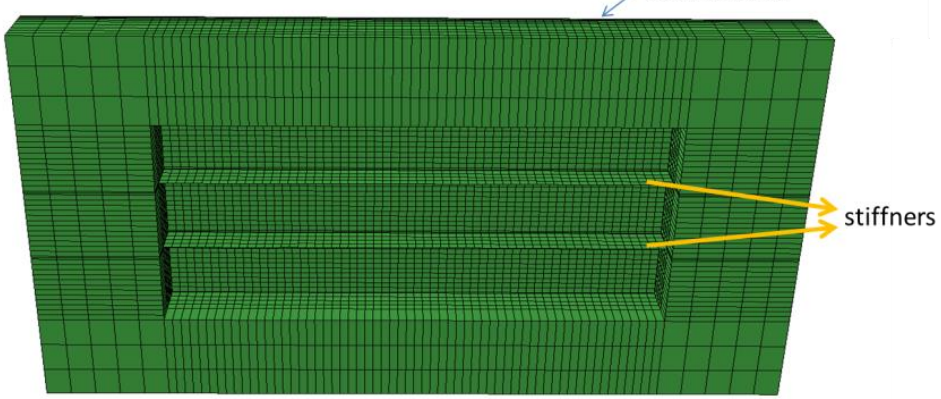

(a) with stiffeners

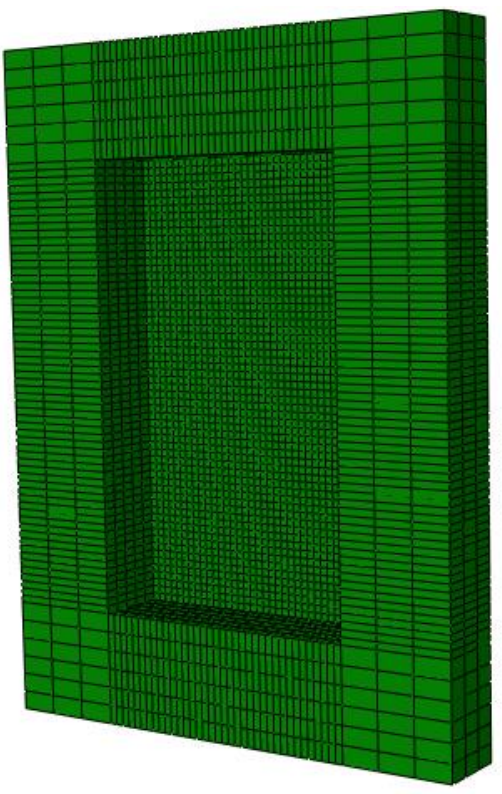

(b) without stiffener

Figure 15 FEA mesh of the indentation tests (a) with stiffener and (b) without stiffener

Fig. 16 shows the computational model associated with the coarse mesh along with its fringe plot for the vertical displacement. A comparison of the predicted deformation is shown in Fig. 16 at two instants, before the crack initiation and after the final fracture pattern is formed; the three graphs in each case show respectively: 1) the experimental data reported by Alsos et al. (2009), 2) the simulation results using DYNA3D developed by Northwestern University (NWU), and 3) the simulation results using GEM's XSHELL toolkit. As shown in Fig. 16, the predicted fracture pattern and load deflection curve agree well with both the test data and NWU's simulation results. 
Submission for Publication in International Journal of Impact Engineering (Special Issue for Experimental Testing and Computational Modeling)

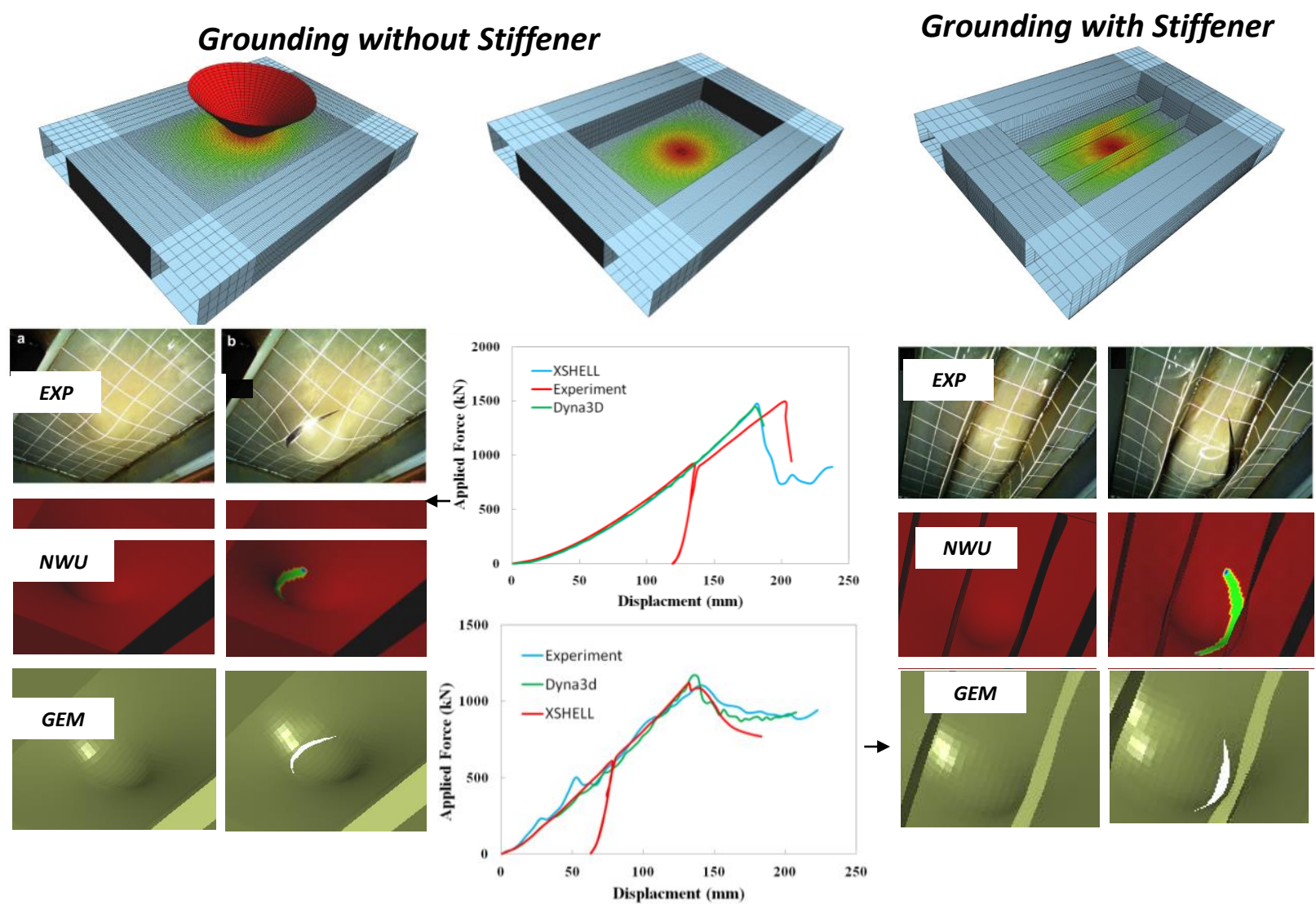

Figure 16 Comparison of GEM's XSHELL prediction of unstiffened and stiffened panels along with its comparison

with the corresponding experimental data and NWU simulation results

\subsection{Crack branching in a five-stringer panel containing a crack}

In order to demonstrate the ability and accuracy of the XSHELL toolkit for simulation of crack branching,

537 a five-stringer panel with a single crack under tension loading (Scheider and Brocks, 2009) is considered here. The geometry of this panel, the crack location and size are shown in Fig. 17. To enhance the computation efficiency, half of the structure is modeled with symmetric boundary conditions. The boundary conditions and FEA mesh are shown in Fig. 18. As we can see in Fig. 18(a), this FEA model is composed

541 with Abaqus S4R elements and XSHELL elements. The XSHELL elements are highlighted in the red color

542 zone, where the rest elements are Abaqus shell elements. The total number of Abaqus S4R elements is 2646 543 and the total number of XSHELL elements is 3871. 
Submission for Publication in International Journal of Impact Engineering (Special Issue for Experimental Testing and Computational Modeling)

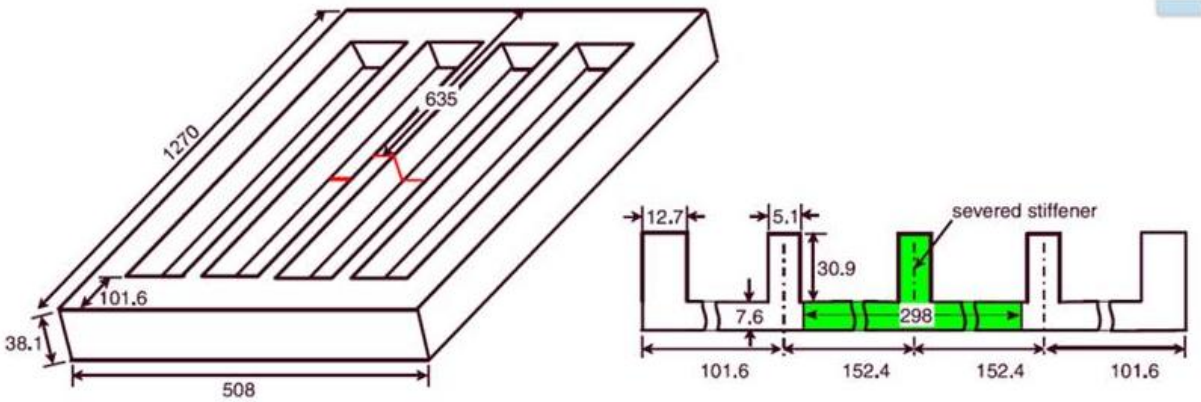

Figure 17 Five-stringer panel containing crack that divides the central stringer (Scheider and Brocks 2009); all dimensions are in $\mathrm{mm}$.
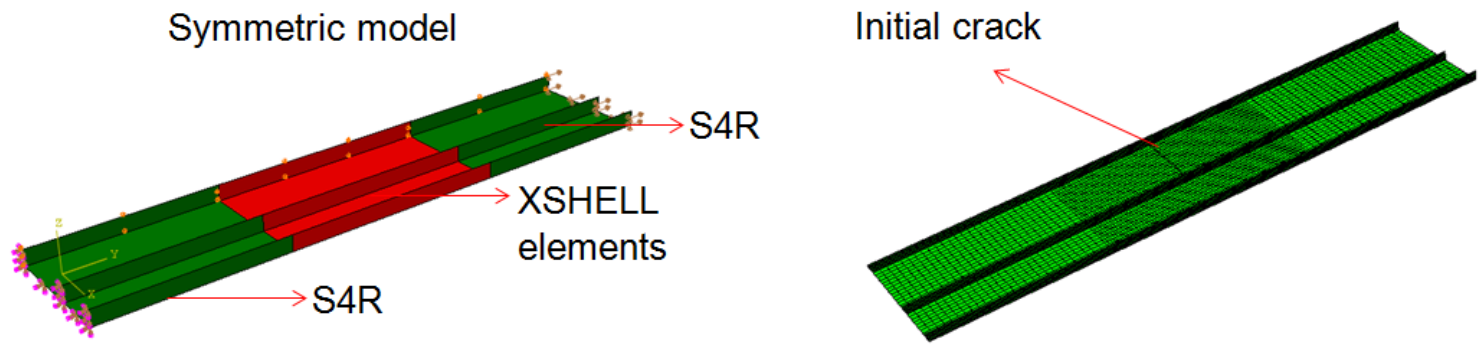

Figure 18 XSHELL Model: (a) FEA model and problem statements; (b) FEA mesh and the location of initial crack.

The material used for this panel is A12024-T351 and its yield strength in the transverse direction, ultimate tensile strength, and its elongation is $342 \mathrm{MPa}, 485 \mathrm{MPa}$, and $18.3 \%$, respectively. Its elastic properties are: elastic modulus $\mathrm{E}=67992 \mathrm{MPa}$, Poisson's ratio $v=0.30$, density $=2.7 e \quad 5 \mathrm{Kg} / \mathrm{m}^{3}$. A $J_{2}$ plasticity with a constant accumulative plastic strain of 0.022 is used for the simulation of crack initiation while the same cohesive properties used by Scheider and Brocks (2009) is used here. The fracture toughness is $11 \mathrm{~kJ} / \mathrm{m}^{2}$ and the maximum cohesive traction is $770 M P a$.

Fig. 19 shows the cracked panel with effective plastic strain contour after the crack has propagated at different loading time; note that the crack has completely cut through the stiffened pane at final loading stage. Crack branching phenomena occurs when the crack tip reaches the stiffener, and our FEA has 
Submission for Publication in International Journal of Impact Engineering (Special Issue for Experimental Testing and Computational Modeling)

successfully simulate the crack turning into the vertical stiffener. The applied stress vs. crack opening displacement (COD) curve is compared with the experimental testing results in Fig. 20. Our numerical prediction agrees well with the experimental data.

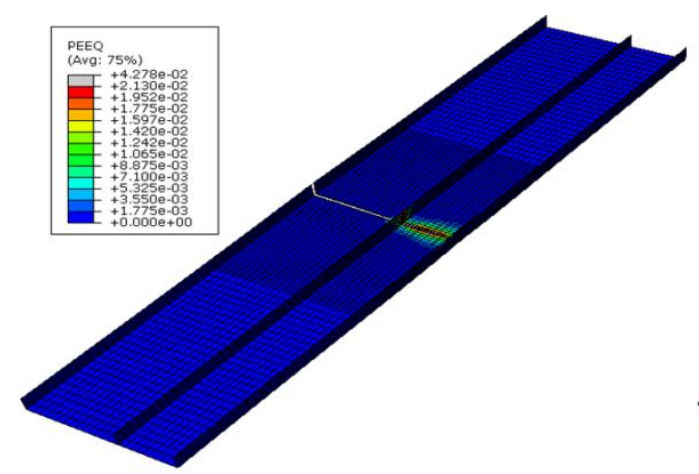

(a)

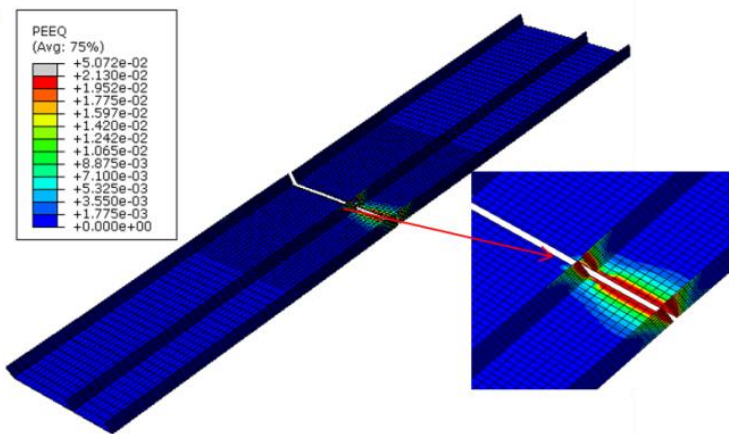

(b)

Figure 19 Effective plastic strain contour on cracked panel at different loading time (a) $t=0.86 \mathrm{~s}$ and (b) $t=0.98 \mathrm{~s}$.

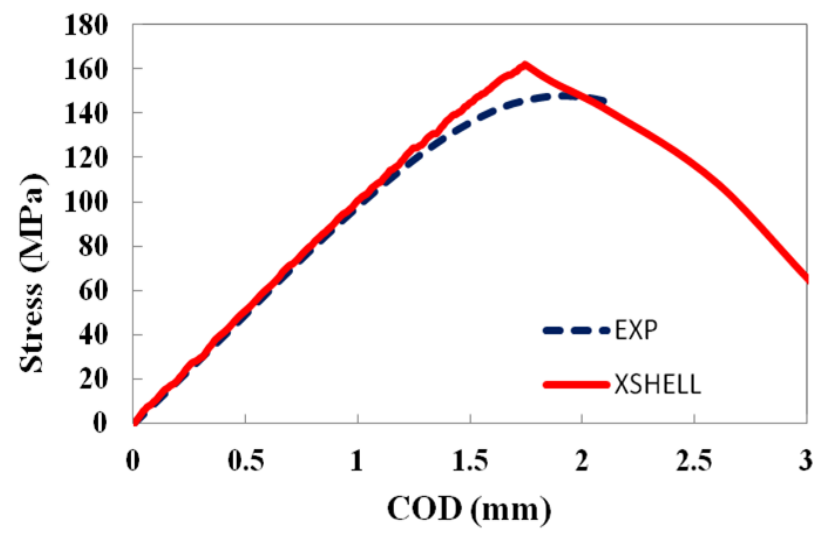

Figure 20 Remote stress vs. COD plot of the stiffened pane

\subsection{Simulation of an explosively loaded square plate}

A sequential coupling has been applied for the dynamic response prediction of a metallic component subjected to an explosive loading. An Abaqus analysis is first performed using its CONWEP loading module and the time history pressure profiles are created for all loaded elements. A load mapping module 
Submission for Publication in International Journal of Impact Engineering (Special Issue for Experimental Testing and Computational Modeling)

577 has been developed to convert the pressure time history to XSHELL pressure loadings. An XSHELL

578 analysis can then be performed using the created loading scenario. A Python script has been developed to

579 convert the force output from an Abaqus simulation into the proper format for XSHELL's pressure loading

580 input. To catch the time history of element forces, multiple data files have first been generated based on an

581 Abaqus simulation under blast wave loading. Each of these files contains the element-based forces

582 outputted at a specific moment. Thus the number of these data files has matched that of data points in load

583 curves to be used by XSHELL.

585 To illustrate the effectiveness of the proposed approach to simulate blast loading with XSHELL, a

586 benchmark example from Abaqus Example Problems Guide was selected. This example simulates a 610

$587 \mathrm{~mm}$ by $610 \mathrm{~mm}$ steel plate with a thickness of $12.7 \mathrm{~mm}$ with a $1 \mathrm{~kg}$ TNT blast load positioned at $105 \mathrm{~mm}$

588 in front of the center of the plate. The four boundary edges are totally clapped. The criterion of accumulated

589 plastic strain is used to propagate the cracks. A small hole of size $10 \mathrm{~mm}$ at the center is introduced in order

590 to simulate multiple crack initiation and propagation along the radial direction as shown in Fig. 21. In order

591 to explore the initial crack configuration on the final rupture pattern, sixteen (16) small cracks of size of

$5920.93 \mathrm{~mm}$ are introduced and uniformly distributed along the hole edge along its radial direction. For 593 comparison, a local mesh without an initial crack is also displayed in Fig. 21.

XSHELL mesh

with a center hole

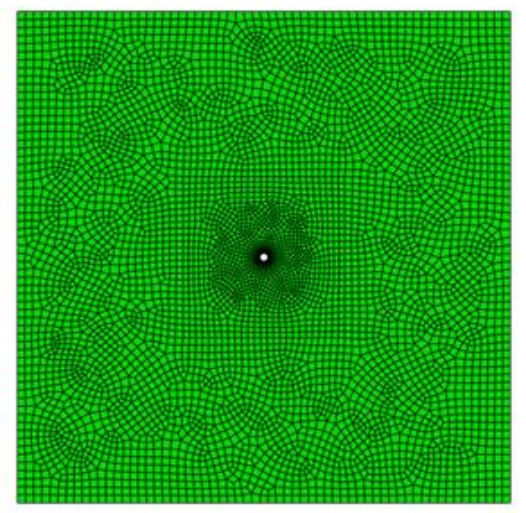

XSHELL mesh with 16 initial cracks

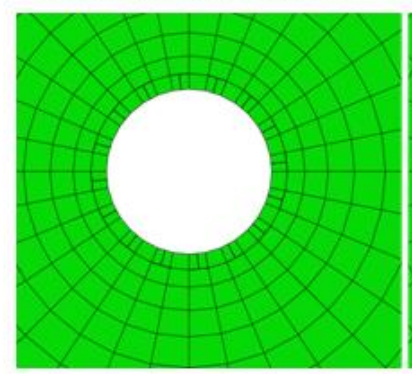

XSHELL mesh without initial cracks

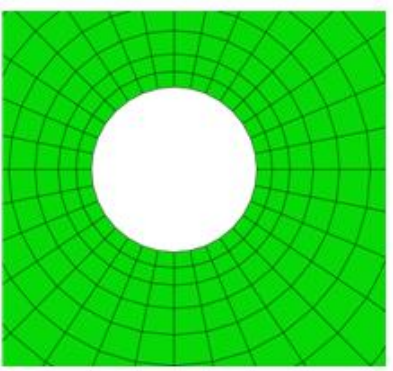


Submission for Publication in International Journal of Impact Engineering (Special Issue for Experimental Testing and Computational Modeling)

Figure 21 Illustration of XSHELL mesh: a) base mesh; 2) mesh with an initial 16 cracks emanating from the hole; and 3) mesh without initial crack

600 Simulation snapshots are illustrated on Fig. 22, which has shown four different moments from the very 601 beginning when an explosive loading is applied to a later stage when the cracks have rapidly propagated. 602 Simulation results with sixteen initial cracks are given in Fig. 22(a) and crack initiation is modeled for the 603 case on Fig. 22(b) where no initial crack is defined. It can be found that both the two cases have shown 604 some similar fracture pattern where the damage at late stage has been dominated by a limited number of 605 fast growing cracks.

606

607

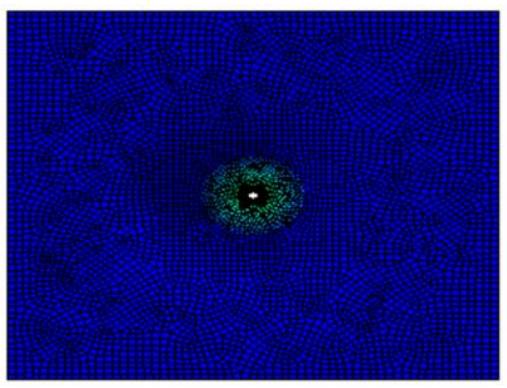

$t=0.00015$

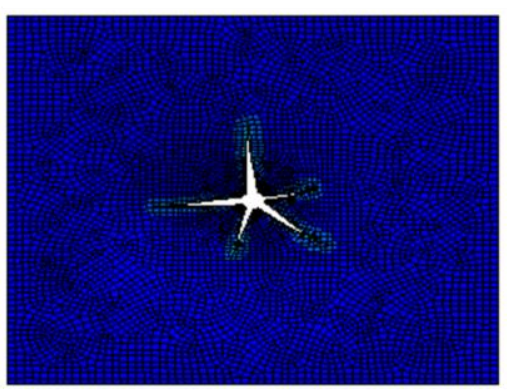

$t=0.00045$

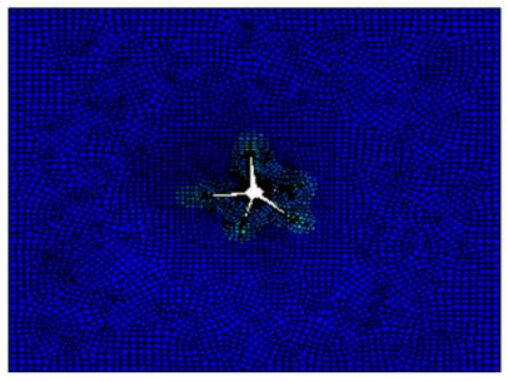

$t=0.00030$

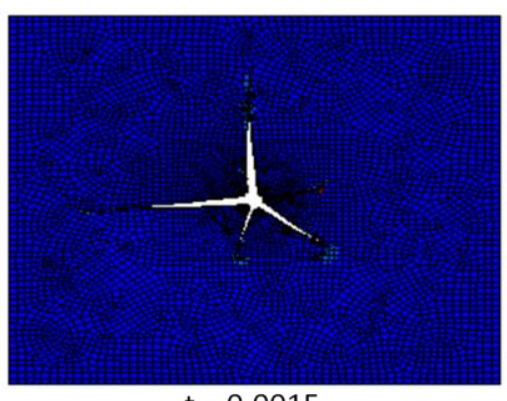

$t=0.0015$

(a) with 16 intial cracks 
Submission for Publication in International Journal of Impact Engineering (Special Issue for Experimental Testing and Computational Modeling)

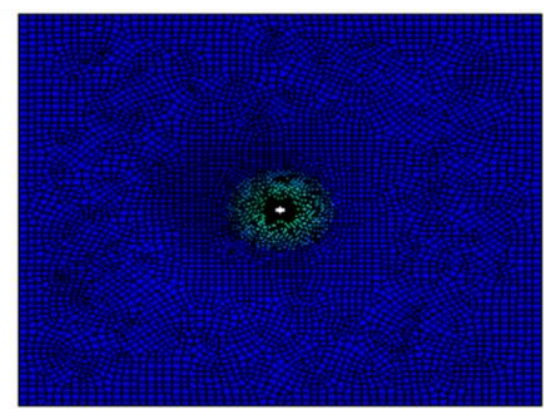

$\mathrm{t}=0.00015$

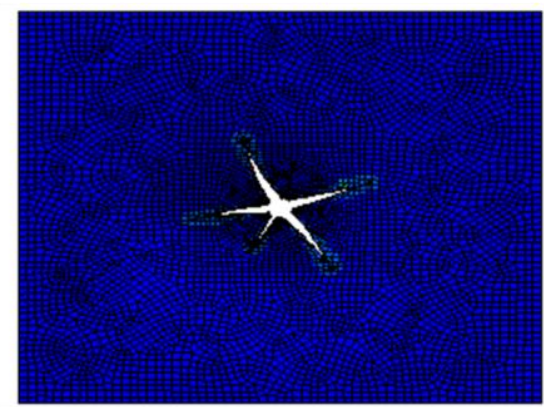

$\mathrm{t}=0.00045$

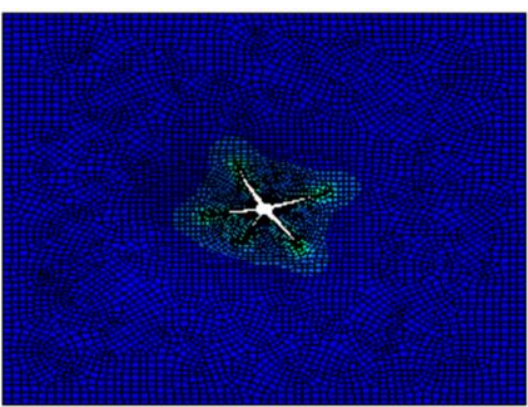

$t=0.00030$

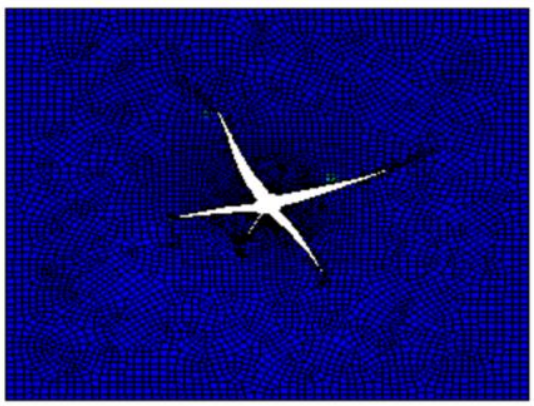

$\mathrm{t}=0.0015$

(b) without initial crack

Figure 22 Simulation example with blast wave loading

\subsection{Hydrostatic and external impulsive pressure induced failure of cylinder}

615 We considered submerged shell structures under impulsive external pressure. For the computations, we 616 considered two types of cylinder structures as shown in Fig. 23; one of the cylinders has two rigid flat ends,

617 and the other has two deformable hemispherical ends. Both cylinders have wall length $\mathrm{L}=8.30 \mathrm{~m}$, wall 618 thickness $\mathrm{t}=0.05 \mathrm{~m}$, and diameter $\mathrm{D}=5.20 \mathrm{~m}$. For the cylinder which has the hemispherical ends, the 619 thickness of the top and the bottom hemispheres are the same as the wall thickness. 


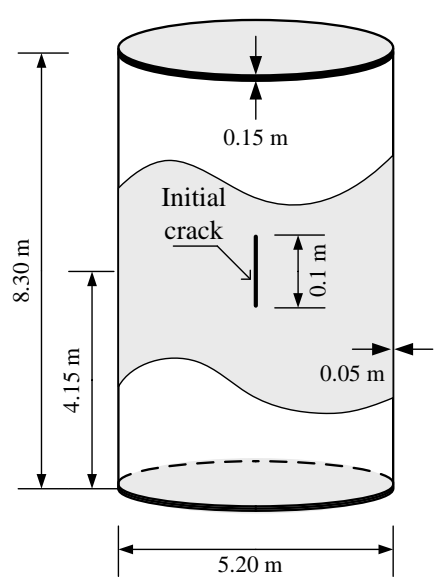

(a)

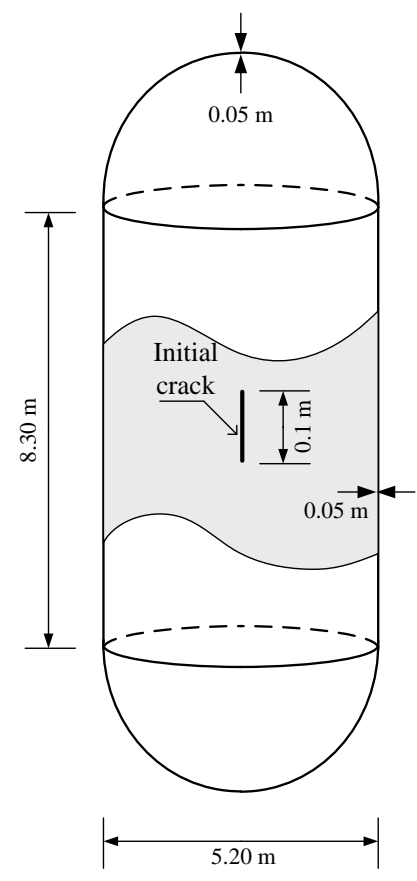

(b)

622 Figure 23 The initial setup of the two cylinders under implosive and impulsive pressures: (a) the cylinder with two rigid flat ends, and (b) the cylinder with two hemisphere ends.

625 For the pressure loading, we superposed implosive hydrostatic pressure along with impulsive external 626 pressure. The time histories of the applied hydrostatic and impulsive pressures are shown in Figs. 24(a) and 627 (b), respectively. The impulsive external pressure is applied to one-side of each cylinder and distributed as a piecewise constant pressure along the circumferential direction as shown in Fig. 25. 
Submission for Publication in International Journal of Impact Engineering (Special Issue for Experimental Testing and Computational Modeling)

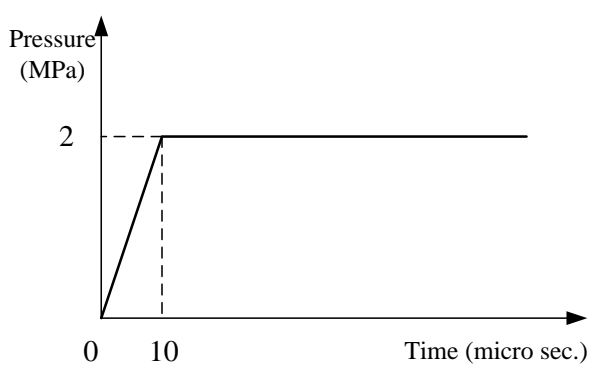

(a)

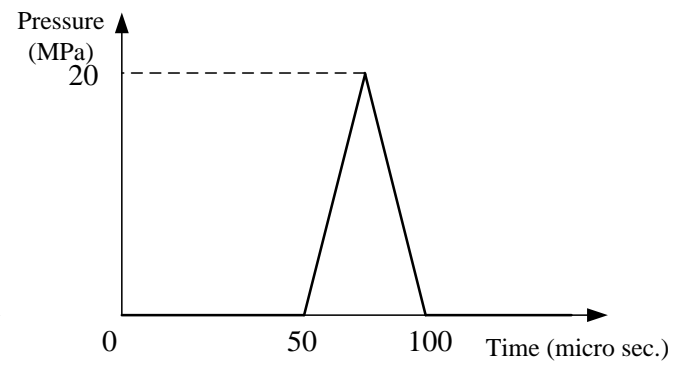

(b)

Figure 24 Time histories of the applied: (a) implosive hydrostatic, and (b) impulsive external pressures.
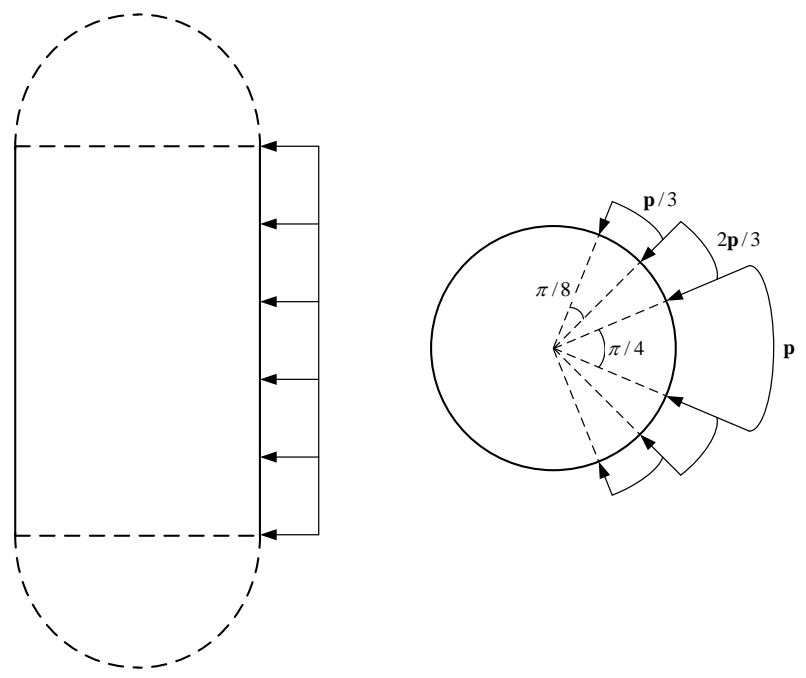

Figure 25 Distribution of the piecewise constant impulsive pressure; the impulsive pressure is only applied to the one side of each cylinder surface.

637 The material is AH36 steel and its material properties are: elastic modulus E $=210 \mathrm{MPa}$, Poisson's ratio $v=0.30$, density $\rho=7850 \mathrm{Kg} / \mathrm{m}^{3}$, and yield stress $\sigma_{y}=355 \mathrm{MPa}$. The shell structures which have the rigid flat ends and the deformable hemispherical ends are modeled with 12,848 and 20,388 4-node quadrilateral shell elements, respectively. Initial notch, $l=0.1 \mathrm{~m}$, is considered at the center of each cylinder, and 0.15

641 effective plastic strain is used for the fracture strain. 
Submission for Publication in International Journal of Impact Engineering (Special Issue for Experimental Testing and Computational Modeling)

642

643 As shown in Fig. 26, the rigid end cap has a significant effect on the crack path. The crack initially forms

644 at the initial notch and then propagates toward the end caps parallel to the axis of the cylinder. However, as

645 the crack tips approach the rigid end caps, the crack tip stresses develop a strong shear component which

646 causes a sudden rotation of the crack trajectory.

647

648 However, for the shell structures with the deformable hemispherical ends, we observed a straight crack

649 growth without any disturbance due to boundary effects. The final crack path and the zoom around the end 650 cap are shown in Figs. 27(c) and 27(d), respectively. During the simulations, due to the applied hydrostatic

651 and impulsive pressures, the cylinder wall deformed toward the inside of the cylinder, and caused the crack 652 surface overlapping. 
Submission for Publication in International Journal of Impact Engineering (Special Issue for Experimental Testing and Computational Modeling)

653

654

655

656

657

658

659

660

661

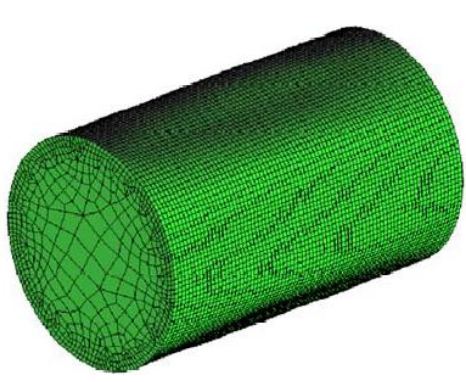

(a)

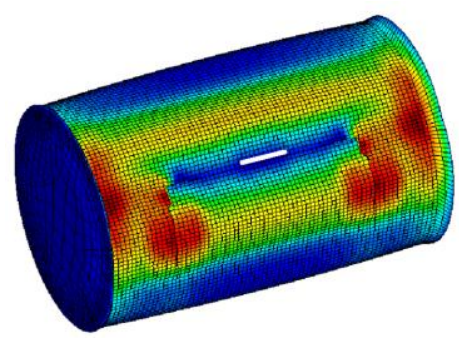

(c)

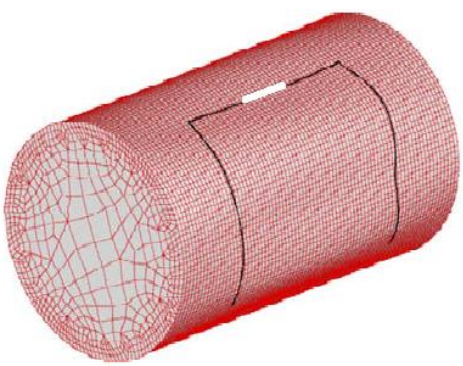

(b)

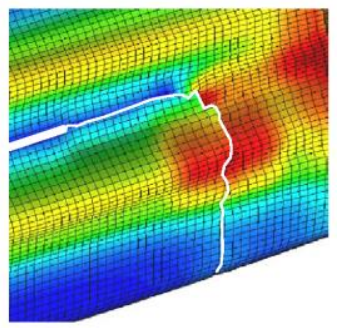

(d)

Figure 26 The simulation results of the shell with two rigid flat ends: (a) the initial mesh which consisted of 12,848 shell elements, (b) representation of the final crack path in the initial configuration, (c) the distribution of effective stress, and (d) zoom around the rotated crack path.

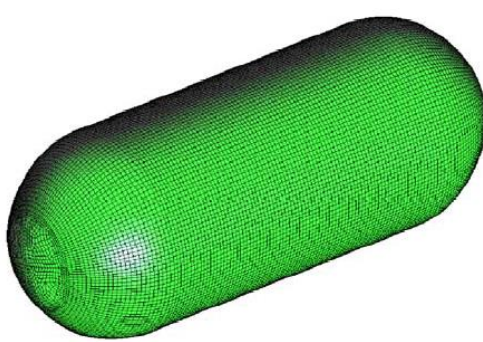

(a)

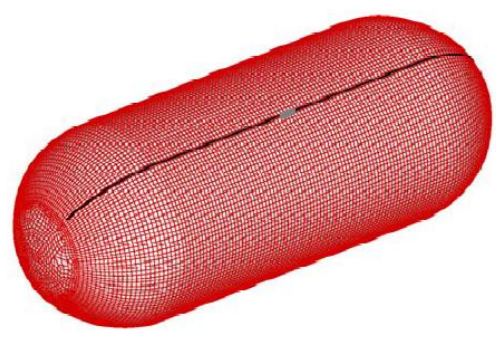

(b) 

in the ductile failure prediction of a global structure.

\section{Summary and Conclusion}

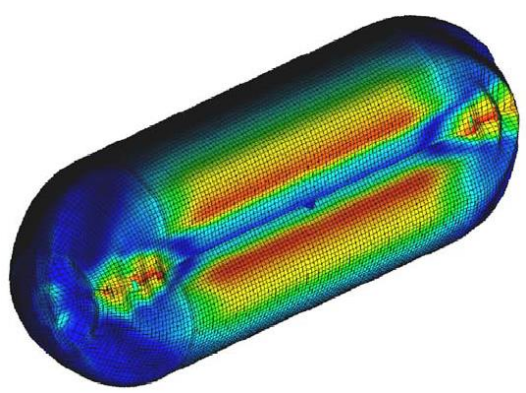

(c)

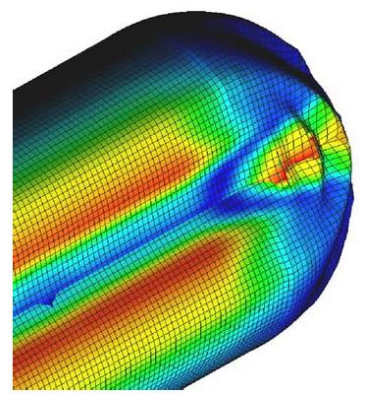

(d)

Figure 27 The simulation results of the shell with two deformable hemispherical ends: (a) the initial mesh which consisted of 20,388 shell elements, (b) representation of the final crack path in the initial domain, (c) the distribution of effective stress, and (d) zoom around the end of crack.

An extended finite element methodology for shell elements has been implemented in Abaqus explicit solver via its VUEL. The resulting XSHELL toolkit can be used for the prediction of the crack path in a large scale thin-walled structure and its associated load-deflection curve. The primary focus of our modeling approach is to capture the dynamics response and ductile failure of a large scale vehicle structure in terms of a correct kinematic description of multiple crack paths, a rational characterization of crack progression and its associated energy dissipation, and an accurate global response prediction in terms of its load deflection behavior. With this mind setting, the XSHELL toolkit couples advanced element technology with widely used phenomenological material and failure models. In particular, to bridge the gap between the local plasticity induced damage initiation and ductile fracture at the structural level, iterative solutions have to be performed to incorporate a length scale in the phenomenological model via a mesh size dependent calibration of failure parameters. The same mesh size during the coupon level calibration is used 
Submission for Publication in International Journal of Impact Engineering (Special Issue for Experimental Testing and Computational Modeling)

682

683

684

685

686

687

688

689

690

691

692

693

694

695

696

697

698

699

700

701

702

703

704

705

706

707

A kinematic description of an arbitrarily cracked shell has been accomplished via the implementation of phantom paired B-T shell elements. A pseudo 8-node user-defined element is pre-defined over a region where a crack initiation and propagation event will occur. During the crack growth simulation, this 8-node shell element has been broken into two 4-node elements through the reconstruction of the element connectivity and the re-distribution of nodal mass associated with two physical nodes and two phantom nodes. A cohesive interaction has been injected on a newly cracked surface to account for the energy dissipation during the crack propagation.

In order to characterize the crack branching that is a frequently met phenomenon during crack propagation in a welded stiffened panel, a special numerical algorithm has been developed for re-grouping and identifying new tip elements when it has met the intersection of multi-components (weld line). Its performance has been demonstrated via the application to a multi-bay stiffened panel with a pre-cracked stiffener.

Despite the use of a conventional J2 plasticity model and a constant accumulative plastic strain criterion for the crack initiation prediction, the global response and final failure pattern have been captured well for the selected example problems including indentation of stiffened and non-stiffened panels, a multi-bay stiffened panel under an axial loading, an explosively loaded square plate, and a cylinder subjected to an internal gaseous detonation. The effects of 3D stress state at the crack tip and the triaxility dependent damage evolution have been studied recently by Zhang et al. (2014) through the application of XSHELL toolkit for modeling and simulation of 2012 Sandia fracture challenge problem (Boyce et al. (2013)).

\section{Acknowledgements}

The authors are grateful for the support provided by the Office of naval Research (N00014-11-C-0487) for which Dr. Paul Hess and Dr. Ken Nahshon serve as the technical monitors. 
Submission for Publication in International Journal of Impact Engineering (Special Issue for Experimental Testing and Computational Modeling)

\section{References}

Amiri F., Milan D., Shen Y., Rabczuk T., Arroyo M. (2014). Phase-field modeling of fracture in linear thin shells, Theoretical and Applied Fracture Mechanics 69, 102.

Areias PMA, Song JH, Belytschko T (2005) A finite-strain quadrilateral shell element based on discrete Kirchhoff-Love constraints. International Journal for Numerical Methods in Engineering 2005 64:1166-1206.

Areias P., Rabczuk T. (2013). Finite strain fracture of plates and shells with configurational forces and edge rotation, International Journal for Numerical Methods in Engineering, 94(12), 1099-1122

Bai Y, Wierzbicki T (2008). A new model of metal plasticity and fracture with pressure and Lode dependence. International Journal of Plasticity. 24, 1071-1096.

Boyce B.L., Kramer S.L.B., Fang H.E. et al. (2013) The Sandia Fracture Challenge: blind round robin predictions of ductile tearing. International Journal of Fracture, 186, 5-68.

Camacho GT, Ortiz M (1996). Computational modelling of impact damage in brittle materials. International Journal of solids and structures,33,2899-2938.

Chau-Dinh T., Zi G., Lee P.S., Song J.H., Rabczuk T. (2013). Phantom-node Method for Shell Models with Arbitrary Cracks, Computers \& Structures, 92-93, 242-256.

Gurson, A.L., (1977). Continuum of ductile rupture by void nucleation and growth: part I. Yield criteria and flow rules for porous ductile media. Journal of Engineering Materials and Technology. 99, 2-55.

Gao X, Zhang T, Hayden M, Roe C (2009) Effects of the stress state on plasticity and ductile failure of an aluminum 5083 alloy. International Journal of Plasticity 25 (12), 2366-2382.

Gao X, Zhang T, Zhou J, Graham SM, Hayden M, Roe C (2011), On Stress-State Dependent Plasticity Modeling: Significance of the Hydrostatic stress, the Third Invariant of Stress Deviator and the NonAssociated Flow Rule, International Journal of Plasticity, vol. 27, 217-231.

Hansbo A, Hansbo P (2004). A finite element method for the simulation of strong and weak discontinuities in solid mechanics. Computer Methods in Applied Mechanics and Engineering 2004; 193:3523-3540. 
Submission for Publication in International Journal of Impact Engineering (Special Issue for Experimental Testing and Computational Modeling)

Johnson G R, Cook W H, (1985). Fracture characteristics of three metals subjected to various strains, strain rates, temperatures and pressures. Engineering Fracture Mechanics. 21, 31-48.

Larsson R, Mediavilla J, Fagerström M (2011). Dynamic fracture modeling in shell structures based on XFEM. International Journal for Numerical Methods in Engineering 86:499-527.

Nahshon K, Hutchinson J W (2008). Modification of the Gurson Model for shear failure European Journal of Mechanics - A/Solids Volume 27, Issue 1, Pages 1-17.

Nguyen-Thanh N., Valizadeh N., Nguyen M.N., Nguyen-Xuan H., Zhuang X. , Areias P., Zi G., Bazilevs Y., De Lorenzis L., Rabczuk T. (2015). An extended isogeometric thin shell analysis based on Kirchhoff-Love theory, Computer Methods in Applied Mechanics and Engineering, 284, 265 - 291.

Rabczuk T., Areias P.M.A. (2006). A meshfree thin shell for arbitrary evolving cracks based on an external enrichment, CMES-Computer Modeling in Engineering and Sciences, 16(2), 115-130.

Rabczuk T., Areias P.M.A., Belytschko T. (2007). A meshfree thin shell method for nonlinear dynamic fracture, International Journal for Numerical Methods in Engineering, 72(5), 524-548.

Rabczuk T., Gracie R., Song J.H., Belytschko T. (2010). Immersed particle method for fluid-structure interaction, International Journal for Numerical Methods in Engineering, 81(1), 48-71.

Scheider I and Brocks Wolfgang (2009). Residual strength prediction of a complex structure using crack extension analyses. Engineering Fracture Mechanics 76, 149-163.

Song J H, Areias PMA, Ted Belytschko (2006). A method for dynamic crack and shear band propagation with phantom nodes. International Journal for Numerical Methods in Engineering 2006; 67:868-893.

Song J H and Belytschko T (2009). Dynamic Fracture of Shells Subjected to Impulsive Loads. Journal of Applied Mechanics 76(5), 051301.

Tvergaard V. and Needleman A. (1984) Analysis of the cup-cone fracture in a round tensile bar. Acta Metallurgica Volume 32, Issue 1, Pages 157-169.

Xu XP, Needleman A (1994) Numerical simulations of fast crack growth in brittle solids. Journal of the Mechanics and Physics of Solids, 42, 1397-1434. 
Submission for Publication in International Journal of Impact Engineering (Special Issue for Experimental Testing and Computational Modeling)

758 Xue L, Tierzbicki T (2009). Numerical simulation of fracture mode transition in ductile plates. 759 International Journal of Solids and Structures 46 (2009) 1423-1435.

760 Zhang T., Fang E., Liu P., and Lua J (2014). Modeling and simulation of 2012 Sandia fracture challenge 761 problems: phantom paired shell for Abaqus and plane strain core approach. Int. J. Fract, 186, 117-139. 